\title{
Integral field spectroscopy of a sample of nearby galaxies
}

\section{Sample, observations, and data reduction ${ }^{\star}$}

\author{
E. Mármol-Queraltón ${ }^{1,2}$, S. F. Sánchez ${ }^{1}$, R. A. Marino ${ }^{1,2}$, D. Mast ${ }^{1,3}$, K. Viironen ${ }^{1,4}$, A. Gil de Paz ${ }^{2}$, \\ J. Iglesias-Páramo ${ }^{3,1}$, F. F. Rosales-Ortega ${ }^{5,1}$, and J. M. Vilchez ${ }^{3}$
}

\author{
1 Centro Astronómico Hispano Alemán, Calar Alto, (CSIC-MPG), C/Jesús Durbán Remón 2-2, 04004 Almería, Spain \\ e-mail: emq@astrax.fis.ucm.es \\ 2 Departamento de Astrofísica y CC. de la Atmósfera, Universidad Complutense de Madrid, 28040-Madrid, Spain \\ 3 Instituto de Astrofísica de Andalucía (CSIC), Camino Bajo de Huetor s/n, Aptdo. 3004, 18080-Granada, Spain \\ ${ }^{4}$ Centro de Estudios de Física del Cosmos de Aragón (CEFCA), C/ Pizarro 1, $3^{a}, 41001$ Teruel, Spain \\ 5 Departamento de Física Teórica, Universidad Autóoma de Madrid, 28049-Cantoblanco, Madrid, Spain
}

Received 5 April 2011 / Accepted 17 June 2011

\section{ABSTRACT}

\begin{abstract}
Aims. Integral field spectroscopy (IFS) is a powerful approach to studying nearby galaxies since it enables a detailed analysis of their resolved physical properties. Here we present our study of a sample of nearby galaxies selected to exploit the two-dimensional information provided by the IFS.

Methods. We observed a sample of 48 galaxies from the local universe with the PPaK integral field spectroscopy unit (IFU), of the PMAS spectrograph, mounted at the $3.5 \mathrm{~m}$ telescope at Calar Alto Observatory (Almeria, Spain). Two different setups were used during these studies (low - V300 - and medium - V600 - resolution mode) covering a spectral range of around 3700-7000 Å . We developed a full automatic pipeline for the data reduction, which includes an analysis of the quality of the final data products. We applied a decoupling method to obtain the ionised gas and stellar content of these galaxies, and derive the main physical properties of the galaxies. To assess the accuracy in the measurements of the different parameters, we performed a set of simulations to derive the expected relative errors obtained with these data. In addition, we extracted spectra for two types of aperture, one central and another integrated over the entire galaxy, from the datacubes. The main properties of the stellar populations and ionised gas of these galaxies and an estimate of their relative errors are derived from those spectra, as well as from the whole datacubes.

Results. We compare the central spectrum extracted from our datacubes and the SDSS spectrum for each of the galaxies for which this is possible, and find close agreement between the derived values for both samples. We find differences on the properties of galaxies when comparing a central and an integrated spectra, showing the effects of the extracted aperture on the interpretation of the data. Finally, we present two-dimensional maps of some of the main properties derived with the decoupling procedure.
\end{abstract}

Key words. surveys - techniques: spectroscopic - galaxies: abundances - stars: formation - galaxies: ISM - galaxies: stellar content

\section{Introduction}

Galaxies in the local universe represent the final products of their cosmological evolution process, and their properties are a direct consequence of their assembly, star formation, metal and gas enrichment, and environmental effects (interactions/mergers). Since all of these processes are imprinted in the spectral properties across their visible surface, a two-dimensional analysis (hereafter 2D) can help us to understand the evolution that galaxies have undergone. The use of integral field spectroscopy (IFS) enables us to build 2D maps and performe a detailed characterisation of their spectroscopic properties, hence IFS is a powerful tool for the study of nearby galaxies.

We present an exploratory study of 48 nearby galaxies observed using the PMAS spectrograph (Postdam Multi Aperture Spectrograph, Roth et al. 2005) in the PPaK mode (PMAS fiber Package Verheijen et al. 2004; Roth et al. 2005), mounted at the $3.5 \mathrm{~m}$ telescope of the Centro Astronómico Hispano

* Based on observations collected at the Centro Astronómico Hispano Alemán (CAHA) at Calar Alto, operated jointly by the MaxPlanck Institut für Astronomie and the Instituto de Astrofísica de Andalucía (CSIC).
Alemán (CAHA, Spain). The wide field-of-view (FoV) of PPaK $\left(74^{\prime \prime} \times 65^{\prime \prime}\right)$ allows us to acquire observations of galaxies in the local universe covering most of their optical extent. We explored two different setups (low and medium spectral resolution) to finally obtain $\sim 40000$ spectra, covering an area of $\sim 50 \mathrm{arcmin}^{2}$. To fully realise the capabilities of this instrument, we observed galaxies in a redshift range of $0.005<z<0.025$, in a compromise between the covered area and their sampling (spatial resolution). This redshift range corresponds to a luminosity distances of $D_{\mathrm{L}}=19-106 \mathrm{Mpc}$, and one arcsec corresponds to a linear scale of $\sim 92-527 \mathrm{pc}$, when assuming a standard $\Lambda \mathrm{CDM}$ cosmology $\left(H_{0}=70.5, \Omega=0.27, \Lambda=0.73\right.$, Hinshaw et al. 2009). Thus, this study enables us to distinguish structures in the galaxies (arms, bars, and bulge/disk), although smaller regions of interest, such as individual H II regions, are not resolved with these observations.

Previous works have explored the use of different integral field spectrophotometers for a detailed study of nearby galaxies. In particular, the SAURON project (Bacon et al. 2001; de Zeeuw et al. 2002) and its extension Atlas3D (Cappellari et al. 2011) are focused on the analysis of early-type galaxies and bulges of spirals at $z<0.01$, to study their kinematics and 
stellar populations. Owing to the distance of their targeted objects $\left(D_{\mathrm{L}}<42 \mathrm{Mpc}\right)$ and the FoV covered by the SAURON instrument $\left(33^{\prime \prime} \times 41^{\prime \prime}\right)$, this study is mainly restricted to the central part of galaxies. The study of nearby spiral galaxies has been addressed by the VENGA survey (32 nearby spiral galaxies, Blanc et al. 2010) using the VIRUS-P spectrograph (Hill et al. 2008), the DiskMass Survey (Bershady et al. 2010a, 146 nearly faceon galaxies) combining PPaK and the SparsePak spectrograph (Bershady et al. 2004, 2005), and the PINGS survey (17 nearby disky galaxies, Rosales-Ortega et al. 2010), using PPaK, as in this work. In particular, the mosaicking designed for the PINGS survey has allowed them to map the H II regions across the whole physical extent of galaxies and explore the 2D metallicity structure of disks. Our sample comprises galaxies of different morphological types in the considered redshift range, with a predominance of spiral galaxies. In addition, they were selected in size to fit the FoV of the PPaK instrument, hence map their physical properties across their whole extension in just one pointing. The results obtained from the PING survey can be compared with this article to analyse distance effects when determining and interpretating their physical properties.

The sample selection, observational strategy, data reduction, and assessment of the accuracy of the final data set presented here formed part of the exploratory studies for the final selection for the optimal instrumental setup and sample of the CALIFA survey (Calar Alto Legacy Integral field spectroscopy Area survey ${ }^{1}$, Sánchez et al. 2010, 2011a). CALIFA is an ongoing observing survey of about 600 nearby galaxies to explore the mechanisms driving their evolution along the colour-magnitude diagram by studying their kinematics, ionised gas, and stellar content. As part of this work, we performed a wide set of simulations to check the quality of the data recovery the physical properties of the observed galaxies. Although this analysis has provided a mark bench in terms of the observational strategy and data reduction for the study of future samples, e.g. the CALIFA survey, the data described in this article constitute an independent data set, which contains interesting physical information about the considered galaxies, hence we present them as a separated study.

In this paper, we focus on the sample (Sect. 2), observations (Sect. 3), and data reduction (Sect. 4). We present a first analysis and results from these data, including a detailed study of the accuracy of the derived physical parameters from the simulations, in Sect. 5. An additional analysis of different aspects of galaxies, as well as the properties of the ionised gas, and particular interesting objects will be presented in forthcoming papers.

\section{Sample}

Two different sample selections are explored in this work: (1) galaxies extracted from the SDSS DR4 imaging sample brighter than $r<15.75$ mag and of redshift $0.005<z<0.025$ (selection in volume and limiting magnitude) and (2) face-on disk galaxies included in the DiskMass Survey (DMS, Bershady et al. 2010a; Verheijen, priv. comm.) with appropriate sizes to fill the FoV of the instrument (angular isophotal-diameter selection). The first criterion is the standard method for selecting galaxies within a redshift range. In this case, the angular size of each galaxy is different, hence the 2D information available for the entire sample is limited. The face-on galaxy sub-sample was selected to help us solve this problem, but the sample is both incomplete and not

\footnotetext{
${ }^{1}$ http://www.caha.es/CALIFA/
}
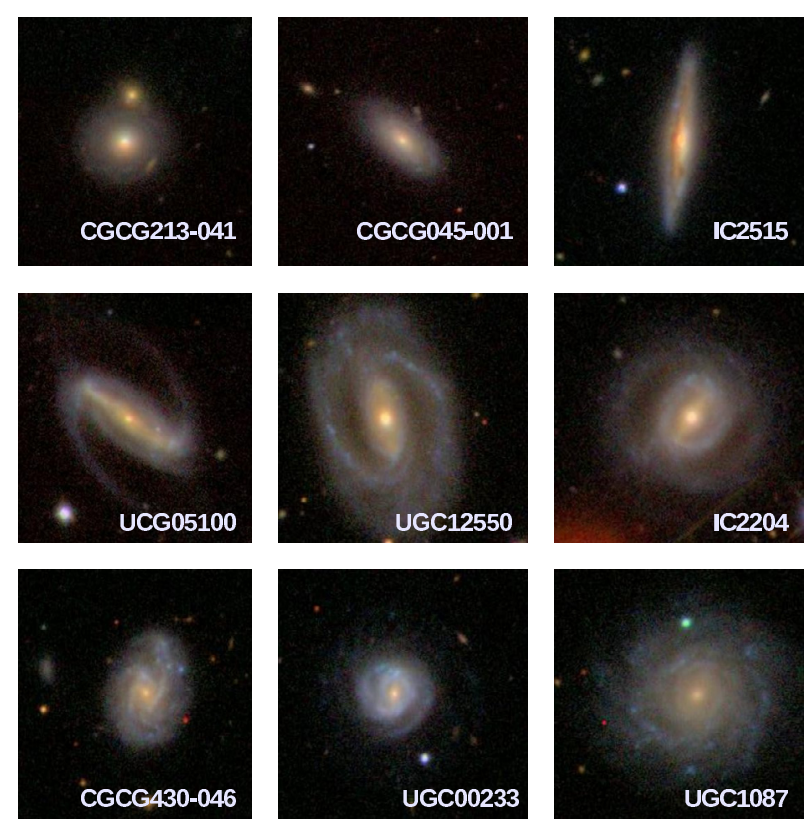

Fig. 1. Subset of nine of the observed galaxies, where the difference in projected sizes can be seen. All images are $1.5^{\prime} \times 1.5^{\prime}$ (north is up, east to left). Images from SDSS survey.

representative of the local universe, and particular care has to be taken when interpreting the results in terms of global evolution.

In total, a subset of 48 galaxies were finally observed, randomly selected from these two samples depending on their visibility during the considered nights. Four galaxies were only partially observed and are not considered in this work. Table 1 lists the observed galaxies, including some basic information such as the name, coordinates, a basic morphological classification (when available), redshift, and $V$-band absolute magnitudes. Although there is a bias towards gas-rich late-type galaxies owing to the selection of the sample, it is a heterogeneous sample that includes bulge-dominated and almost pure-disk galaxies, face-on and edge-on objects, and galaxies with and without bars. This is shown in Fig. 1, where some galaxies included in this study are presented.

Finally, we present in Fig. 2 the colour-magnitude diagram of the observed objects, indicating those ones coming from each sub-sample. Regions corresponding to the red sequence, the green valley, and the blue cloud are also indicated (e.g., Strateva et al. 2001; Bell et al. 2004, 2006; Faber et al. 2007; Chen et al. 2010). Although the sample presented here is small, there is a significant number of observed galaxies in both the red sequence and the blue cloud, as well as the green valley. Therefore, this sample will allow a preliminary study of the global evolution of galaxies in the local universe.

\section{Observations}

Observations were carried out over fifteen nights in several observing runs. Information about the weather conditions during the observations are presented in Table 2. The full sample was observed at the $3.5 \mathrm{~m}$ telescope of the Calar Alto observatory using the PMAS spectrograph (Postdam Multi Aperture Spectrograph, Roth et al. 2005) in the PPaK mode (Verheijen et al. 2004; Roth et al. 2005). The PPaK fibre bundle consists of 382 fibres of 2.7" diameter each (see Fig. 5 in Roth et al. 2005). The science fibres (331 fibres) are concentrated in a single hexagonal bundle, with a filling factor of $\sim 65 \%$. The 
E. Mármol-Queraltó et al.: Integral field spectroscopy of a sample of nearby galaxies. I.

Table 1. General information about the observed galaxies.

\begin{tabular}{|c|c|c|c|c|c|c|c|c|c|c|c|}
\hline Galaxy & $\begin{array}{c}\text { RA (J2000) } \\
(2) \\
\end{array}$ & $\begin{array}{c}\text { Dec (J2000) } \\
\text { (3) } \\
\end{array}$ & $\begin{array}{l}\text { Type } \\
\text { (4) } \\
\end{array}$ & $\begin{array}{l}z \\
(5) \\
\end{array}$ & $\begin{array}{c}R_{25} \\
(\mathrm{mag}) \\
(6) \\
\end{array}$ & $\begin{array}{c}M_{V} \\
(\mathrm{mag}) \\
(7) \\
\end{array}$ & $\begin{array}{c}(B-V) \\
(\mathrm{mag}) \\
(8) \\
\end{array}$ & $\begin{array}{c}\text { Observing date } \\
\text { (yy-mm-dd) } \\
(9)\end{array}$ & $\begin{array}{l}\text { Grid } \\
(10) \\
\end{array}$ & $\begin{array}{l}\text { Seeing } \\
\left({ }^{\prime \prime}\right) \\
(11) \\
\end{array}$ & $\begin{array}{c}\begin{array}{c}\text { Airmass } \\
(\mathrm{mag})\end{array} \\
(12) \\
\end{array}$ \\
\hline KUG $1033+403$ & $10^{\mathrm{h}} 36^{\mathrm{m}} 12.4^{s}$ & $+40^{\circ} 03^{\prime} 12.6^{\prime \prime}$ & $\mathrm{S} ?$ & 0.0229 & 0.56 & 16.5 & 0.87 & $2009-03-23$ & V300 & $\ldots$ & 1.12 \\
\hline 2MASX J13062093+5318232† & $13^{\mathrm{h}} 06^{\mathrm{m}} 21.0^{s}$ & $+53^{\circ} 18^{\prime} 23.2^{\prime \prime}$ & AGN & 0.0237 & $\ldots$ & 15.9 & 1.25 & $2009-03-22$ & V300 & 2.0 & 1.11 \\
\hline 2 MASX J13193595+5330102 † & $13^{\mathrm{h}} 19^{\mathrm{m}} 35.9^{s}$ & $+53^{\circ} 30^{\prime} 09.8^{\prime \prime}$ & $\operatorname{IrS}$ & 0.0248 & $\ldots$ & 12.4 & 0.72 & $2009-03-24$ & V300 & 2.0 & 1.13 \\
\hline CGCG 045-001 & $13^{\mathrm{h}} 30^{\mathrm{m}} 43.5^{\mathrm{s}}$ & $+07^{\circ} 31^{\prime} 48.8^{\prime \prime}$ & $\ldots$ & 0.0220 & $\ldots$ & 15.4 & 1.07 & $2009-03-20$ & V300 & 1.3 & 1.19 \\
\hline CGCG 071-096 † & $13^{\mathrm{h}} 00^{\mathrm{m}} 33.2^{s}$ & $+10^{\circ} 07^{\prime} 47.8^{\prime \prime}$ & $\ldots$ & 0.0239 & $\cdots$ & 15.1 & 0.79 & $2009-03-20$ & V300 & 1.1 & 1.25 \\
\hline CGCG $148-006 \dagger$ & $07^{\mathrm{h}} 44^{\mathrm{m}} 57.4^{s}$ & $+28^{\circ} 55^{\prime} 39.0^{\prime \prime}$ & $\ldots$ & 0.0234 & $\ldots$ & 15.3 & 0.75 & $2009-10-23$ & V600 & 1.1 & 1.30 \\
\hline CGCG $181-068 \dagger$ & $09^{\mathrm{h}} 37^{\mathrm{m}} 19.2^{s}$ & $+33^{\circ} 49^{\prime} 25.8^{\prime \prime}$ & $\ldots$ & 0.0227 & $\ldots$ & 15.7 & 1.17 & $2009-03-20$ & V300 & 1.2 & 1.06 \\
\hline CGCG 213-041 † & $11^{\mathrm{h}} 21^{\mathrm{m}} 16.8^{s}$ & $+40^{\circ} 20^{\prime} 43.4^{\prime \prime}$ & $\ldots$ & 0.0208 & $\ldots$ & 15.5 & 1.16 & $2009-03-21$ & V300 & 1.7 & 1.04 \\
\hline CGCG 293-023 $\dagger$ & $02^{\mathrm{h}} 30^{\mathrm{m}} 21.5^{s}$ & $+56^{\circ} 47^{\prime} 29.5^{\prime \prime}$ & $\ldots$ & 0.0156 & $\ldots$ & 15.6 & 0.99 & $2009-03-23$ & V300 & & 1.18 \\
\hline CGCG $428-059 \dagger$ & $22^{\mathrm{h}} 14^{\mathrm{m}} 51.4^{s}$ & $+13^{\circ} 42^{\prime} 54.9^{\prime \prime}$ & $\ldots$ & 0.0242 & $\ldots$ & 15.7 & 0.90 & $2009-06-27$ & V300 & 1.0 & 1.28 \\
\hline CGCG 428-060 & $22^{\mathrm{h}} 14^{\mathrm{m}} 57.9^{s}$ & $+13^{\circ} 45^{\prime} 42.9^{\prime \prime}$ & $\ldots$ & 0.0242 & $\ldots$ & 13.7 & 0.92 & $2009-06-28$ & V300 & 1.2 & 1.21 \\
\hline CGCG $430-046 \dagger$ & $23^{\mathrm{h}} 00^{\mathrm{m}} 46.2^{s}$ & $+13^{\circ} 37^{\prime} 07.9^{\prime \prime}$ & $\ldots$ & 0.0243 & $\ldots$ & 15.3 & 0.86 & $2009-10-23$ & V600 & 1.1 & 1.10 \\
\hline IC $2204 \dagger$ & $07^{\mathrm{h}} 41^{\mathrm{m}} 18.1^{s}$ & $+34^{\circ} 13^{\prime} 55.9^{\prime \prime}$ & (R)SB(r)ab & 0.0155 & 0.00 & 14.6 & 0.93 & $2009-10-22$ & V600 & 1.5 & 1.12 \\
\hline IC $2500 \dagger$ & $09^{\mathrm{h}} 42^{\mathrm{m}} 23.4^{s}$ & $+36^{\circ} 20^{\prime} 59.1^{\prime \prime}$ & $\ldots$ & 0.0221 & $\ldots$ & 15.2 & 1.17 & $2009-03-21$ & V300 & 1.7 & 1.14 \\
\hline IC $2515 \dagger$ & $09^{\mathrm{h}} 54^{\mathrm{m}} 39.4^{s}$ & $+37^{\circ} 24^{\prime} 30.8^{\prime \prime}$ & $\mathrm{Sb}$ & 0.0193 & 0.72 & 15.1 & 1.11 & $2009-03-22$ & V300 & 1.6 & 1.05 \\
\hline MRK & $13^{\mathrm{h}} 16^{\mathrm{m}} 14.7^{s}$ & $+41^{\circ} 29^{\prime} 40.1^{\prime \prime}$ & $\ldots$ & 0.0207 & $\ldots$ & 15.4 & 0.47 & $-03-24$ & V300 & 1.8 & 1.04 \\
\hline NGC $99 \dagger, \star$ & $00^{\mathrm{h}} 23^{\mathrm{m}} 59.5^{s}$ & $+15^{\circ} 46^{\prime} 13.7^{\prime \prime}$ & Scd & 0.0177 & 0.04 & 14.0 & 0.62 & $2009-10-22$ & V600 & 2.3 & 1.11 \\
\hline NGC $3820 \dagger$ & $11^{\mathrm{h}} 42^{\mathrm{m}} 04.9^{s}$ & $+10^{\circ} 23^{\prime} 03.3^{\prime \prime}$ & S? & 0.0203 & 0.17 & 14.9 & 0.95 & $2009-03-22$ & V300 & 1.2 & 1.40 \\
\hline NGC $4109 \dagger \star \star$ & $12^{\mathrm{h}} 06^{\mathrm{m}} 51.1^{s}$ & $+42^{\circ} 59^{\prime} 44.3^{\prime \prime}$ & Sa? & 0.0235 & 0.05 & 14.8 & 0.98 & $2009-03-19$ & V300 & & 1.05 \\
\hline NGC $7570 \dagger, \star$ & $23^{\mathrm{h}} 16^{\mathrm{m}} 44.7^{s}$ & $+13^{\circ} 28^{\prime} 58.8^{\prime \prime}$ & $\mathrm{SBa}$ & 0.0157 & 0.23 & 13.9 & 0.81 & $2009-10-22$ & V600 & 2.0 & 1.13 \\
\hline UGC 74 & $00^{\mathrm{h}} 08^{\mathrm{m}} 44.7^{s}$ & $+04^{\circ} 36^{\prime} 45.1^{\prime \prime}$ & $\mathrm{SAB}(\mathrm{rs}) \mathrm{c} ?$ & 0.0131 & 0.05 & 14.5 & 0.85 & $2009-10-17$ & V600 & 1.3 & 1.23 \\
\hline UGC 233 & $00^{\mathrm{h}} 24^{\mathrm{m}} 42.7^{s}$ & $+14^{\circ} 49^{\prime} 28.8^{\prime \prime}$ & $\mathrm{SBbc} D$ & 0.0176 & 0.00 & 14.6 & 0.62 & $2009-10-23$ & V600 & 1.1 & 1.20 \\
\hline UGC 448 & $00^{\mathrm{h}} 42^{\mathrm{m}} 22.0^{s}$ & $+29^{\circ} 38^{\prime} 29.9^{\prime \prime}$ & $\mathrm{SABc}$ & 0.0162 & 0.04 & 14.4 & 1.16 & $2009-10-19$ & V600 & 2.0 & 1.08 \\
\hline UGC $463 \dagger$ & $00^{\mathrm{h}} 43^{\mathrm{m}} 32.4^{s}$ & $+14^{\circ} 20^{\prime} 33.2^{\prime \prime}$ & $\mathrm{SAB}(\mathrm{rs}) \mathrm{c}$ & 0.0148 & 0.00 & 13.5 & 0.85 & $2009-10-18$ & V600 & 1.8 & 1.29 \\
\hline UGC 1081 & $01^{\mathrm{h}} 30^{\mathrm{m}} 46.6^{s}$ & $+21^{\circ} 26^{\prime} 25.5^{\prime \prime}$ & $\mathrm{SB}(\mathrm{rs}) \mathrm{c}$ & 0.0104 & 0.03 & 13.8 & 0.83 & $2009-10-22$ & V600 & 2.4 & 1.15 \\
\hline UGC $1087 \dagger$ & $01^{\mathrm{h}} 31^{\mathrm{m}} 26.6^{s}$ & $+14^{\circ} 16^{\prime} 39.0^{\prime \prime}$ & $\mathrm{SA}(\mathrm{rs}) \mathrm{c}$ & 0.0149 & 0.03 & 15.1 & 0.69 & $2009-10-17$ & V600 & 1.0 & 1.13 \\
\hline UGC 1529 & $02^{\mathrm{h}} 02^{\mathrm{m}} 31.0^{s}$ & $+11^{\circ} 05^{\prime} 35.1^{\prime \prime}$ & $\mathrm{SA}(\mathrm{rs}) \mathrm{c}$ & 0.0155 & 0.07 & 14.7 & 1.01 & $2009-10-18$ & V600 & 1.5 & 1.17 \\
\hline UGC 1635 & $02^{\mathrm{h}} 08^{\mathrm{m}} 27.7^{s}$ & $+06^{\circ} 23^{\prime} 41.7^{\prime \prime}$ & SAbc & 0.0115 & 0.01 & 14.8 & 0.96 & $2009-10-23$ & V600 & 1.2 & 1.10 \\
\hline UGC 1862 & $02^{\mathrm{h}} 24^{\mathrm{m}} 24.8^{s}$ & $-02^{\circ} 09^{\prime} 44.5^{\prime \prime}$ & SAB(rs)d pec? & 0.0046 & 0.11 & 14.0 & 0.83 & $2009-10-23$ & V600 & 1.3 & 1.20 \\
\hline UGC 3091 & $04^{\mathrm{h}} 33^{\mathrm{m}} 56.1^{s}$ & $+01^{\circ} 06^{\prime} 49.5^{\prime \prime}$ & $\mathrm{SAB}(\mathrm{s}) \mathrm{d}$ ? & 0.0184 & $\ldots$ & 15.4 & 0.88 & $2009-10-17$ & V600 & 0.9 & 1.40 \\
\hline UGC 3140 & $04^{\mathrm{h}} 42^{\mathrm{m}} 54.9^{s}$ & $+00^{\circ} 37^{\prime} 06.9^{\prime \prime}$ & SA(rs)c? & 0.0154 & 0.06 & 13.6 & 0.97 & $2009-10-19$ & V600 & 1.8 & 1.36 \\
\hline UGC 3701 & $07^{\mathrm{h}} 11^{\mathrm{m}} 42.7^{s}$ & $+72^{\circ} 10^{\prime} 09.5^{\prime \prime}$ & SA(rs)cd? & 0.0097 & 0.00 & 15.2 & 0.87 & $2009-10-17$ & V600 & 1.5 & 1.30 \\
\hline 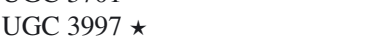 & $07^{\mathrm{h}} 44^{\mathrm{m}} 38.7^{s}$ & $+40^{\circ} 21^{\prime} 58.5^{\prime \prime}$ & Im? & 0.0197 & 0.06 & 16.0 & 1.07 & $2009-10-18$ & V600 & 15 & 1.13 \\
\hline UGC 4036 & $07^{\mathrm{h}} 51^{\mathrm{m}} 54.7^{s}$ & $+73^{\circ} 00^{\prime} 56.5^{\prime \prime}$ & $\mathrm{SAB}(\mathrm{r}) \mathrm{b} ?$ & & 0.06 & 12. & 0.98 & $2009-10-19$ & V600 & 1.6 & 1.38 \\
\hline UGC 4107 & $07^{\mathrm{h}} 57^{\mathrm{m}} 01.9^{s}$ & $+49^{\circ} 34^{\prime} 02.5^{\prime \prime}$ & $\mathrm{SA}(\mathrm{rs}) \mathrm{c}$ & 0.0117 & 0.01 & 13.9 & 1.06 & $2009-10-22$ & V600 & 1.2 & 1.13 \\
\hline UGC $5100 \dagger$ & $09^{\mathrm{h}} 34^{\mathrm{m}} 38.6^{s}$ & $+05^{\circ} 50^{\prime} 29.9^{\prime \prime}$ & $\mathrm{SB}(\mathrm{s}) \mathrm{b}$ & 0.0184 & 0.22 & 14.9 & 1.02 & $2009-03-19$ & V300 & 1.1 & 1.23 \\
\hline UGC $6156 \dagger$ & $11^{\mathrm{h}} 06^{\mathrm{m}} 31.3^{s}$ & $+48^{\circ} 39^{\prime} 05.7^{\prime \prime}$ & S? & 0.0245 & 0.56 & 15.1 & 0.54 & $2009-03-24$ & V300 & 1.5 & 1.11 \\
\hline UGC $6410 \dagger$ & $11^{\mathrm{h}} 24^{\mathrm{m}} 05.9^{s}$ & $+45^{\circ} 48^{\prime} 39.9^{\prime \prime}$ & $\mathrm{SABc}$ & 0.0187 & 0.11 & 14.8 & 0.78 & $2009-03-20$ & V300 & 1.5 & 1.08 \\
\hline UGC $7993 \dagger$ & $12^{\mathrm{h}} 50^{\mathrm{m}} 31.6^{s}$ & $+52^{\circ} 07^{\prime} 22.5^{\prime \prime}$ & Scd? & 0.0161 & 0.90 & 15.3 & 0.79 & $2009-03-19$ & V300 & 1.3 & 1.25 \\
\hline UGC $9837 \dagger$ & $15^{\mathrm{h}} 23^{\mathrm{m}} 51.7^{s}$ & $+58^{\circ} 03^{\prime} 10.6^{\prime \prime}$ & $\mathrm{SAB}(\mathrm{s}) \mathrm{c}$ & 0.0089 & 0.02 & 14.6 & 0.57 & $2007-06-23$ & V300 & $\ldots$ & 1.10 \\
\hline UGC 9965 & $15^{\mathrm{h}} 40^{\mathrm{m}} 06.7^{s}$ & $+20^{\circ} 40^{\prime} 50.2^{\prime \prime}$ & & & 0.06 & 14.4 & 0.72 & $2007-06-22$ & V300 & $\ldots$ & 1.39 \\
\hline UGC 11318 & $18^{\mathrm{h}} 39^{\mathrm{m}} 12.2^{s}$ & $+55^{\circ} 38^{\prime} 30.5^{\prime \prime}$ & $\mathrm{SB}(\mathrm{rs}) \mathrm{bc}$ & 0.0196 & 0.03 & 14.1 & 0.72 & $2007-06-23$ & V300 & $\ldots$ & 1.10 \\
\hline UGC $12250 \dagger$ & $22^{\mathrm{h}} 55^{\mathrm{m}} 35.9^{s}$ & $+12^{\circ} 47^{\prime} 25.1^{\prime \prime}$ & $\mathrm{SBb}$ & 0.0242 & 0.22 & 14.1 & 1.04 & $2009-10-18$ & V600 & 1.1 & 1.33 \\
\hline UGC 12391 & $23^{\mathrm{h}} 08^{\mathrm{m}} 57.2^{s}$ & $+12^{\circ} 02^{\prime} 52.9^{\prime \prime}$ & $\mathrm{SAB}(\mathrm{s}) \mathrm{c}$ & 0.0163 & 0.04 & 14.7 & 0.77 & $2009-10-17$ & V600 & 1.5 & 1.20 \\
\hline
\end{tabular}

Notes. All the galaxies are included in $2 \mathrm{MASS}^{a}$. Galaxies marked with $\dagger$ have available SDSS spectroscopy. Galaxies marked with $\star$ have been observed with GALEX ${ }^{b}$. (1) Galaxy identification; coordinates: (2) right ascension and (3) declination in J2000 Equinox from NED ${ }^{c}$; (4) morphological type from RC3 catalogue ${ }^{d}$; (5) redshift from NED; (6) $R_{25}$ : radius where the surface brightness reaches $\mu_{B}=25.0 \mathrm{mag} / \mathrm{arcsec}^{2}$, obtained from RC3 catalogue; (7) $M_{V}$ : absolute $B$-band magnitude from NED; (8) (B-V); (9) observing date; (10) PPaK mode utilised for each observation (see Table 3); (11) average seeing value during the corresponding night observation. Data from the Calar Alto Observatory archive. (12) airmass for the each observation. ${ }^{(a)}$ Two Micron All Sky Survey (Skrutskie et al. 2006): http://www . ipac . caltech. edu/2mass/. ${ }^{(b)}$ The GALEX Ultraviolet Atlas of Nearby Galaxies (Gil de Paz et al. 2007). ${ }^{(c)}$ NASA/IPAC Extragalactic Database: http: //nedwww . ipac . caltech. edu/. ${ }^{(d)}$ Third Reference Catalogue of Bright Galaxies: http://vizier.u-strasbg.fr/viz-bin/VizieR?-source=VII/155.

sky background is sampled by 36 additional fibres, distributed in 6 bundles of 6 fibres each, distributed along a circle $\sim 90^{\prime \prime}$ from the centre of the instrument FoV. The sky-fibres are distributed among the science fibres within the pseudo-slit to ensure a good characterisation of the sky; the remaining 15 fibres are used for calibration purposes.

A dithering scheme with three pointings was adopted during the different observing runs, to cover the complete FoV of the bundle and increase the spatial resolution of the data. This scheme has already been adopted in previous studies using $\mathrm{PPaK}$, with a considerable increase in the quality of the data (e.g., Sánchez et al. 2007b; Castillo-Morales et al. 2010; Pérez-Gallego et al. 2010; Rosales-Ortega et al. 2010). The offsets in RA-Dec of the different pointings, with respect to the nominal coordinates of the targets, were (in $\operatorname{arcsec})$ : \#1) $(0,0)$; \#2) $(+1.56,+0.78)$; and \#3) $+(1.56,-0.78)$, which allows us to cover the holes between fibres in the central bundle. The spatial re-composition of the three pointings is included in the standard data reduction scheme adopted for these data (described in Sect. 4).

Two different setups were used during these observations, listed in Table 3. For the runs before October 2010, the V300 


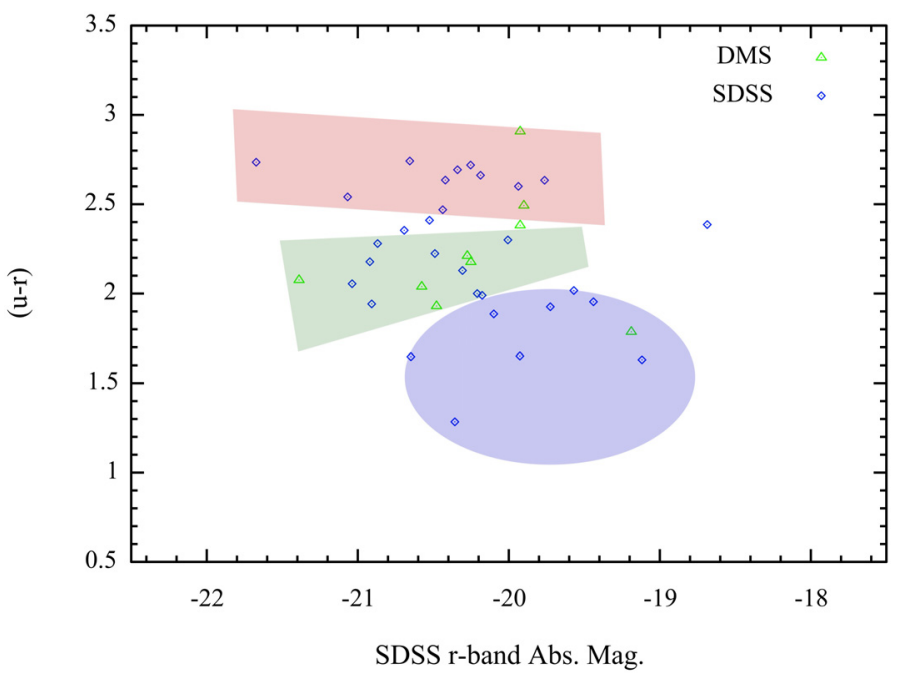

Fig. 2. $u-r$ vs. $M_{r}$ colour-magnitude diagram of the observed sample. In blue circles, we have indicated the DR4 selected sample, and in green triangles those galaxies from the Disk Mass Survey (see text for more detail). The red sequence, green valley, and blue cloud are noted with shaded colour areas. It is clear the observed sample still covers each region with a significant number of objects.

Table 2. Atmospheric conditions during the observations.

\begin{tabular}{llcccc}
\hline $\begin{array}{l}\text { Observing } \\
\text { date }\end{array}$ & & $\begin{array}{c}\text { Seeing } \\
\left({ }^{\prime \prime}\right)\end{array}$ & $\begin{array}{c}\text { Humidity } \\
(\%)\end{array}$ & $\begin{array}{c}\text { Tranparency } \\
(\%)\end{array}$ & $\begin{array}{c}\text { Extinction } \\
\text { in } V \text {-band (mag) }\end{array}$ \\
\hline $2007-06-22^{a}$ & Clear & - & 56 & 95 & 0.22 \\
$2007-06-23^{a}$ & Clear & - & 48 & 95 & 0.22 \\
$2009-03-19$ & Clear & 1.2 & 43 & 95 & 0.15 \\
$2009-03-20$ & Clear & 1.3 & 62 & 85 & 0.25 \\
$2009-03-21$ & Clouds & 1.7 & 71 & 75 & 0.28 \\
$2009-03-22$ & Clear & 2.0 & 70 & 95 & 0.15 \\
$2009-03-23^{a}$ & Clouds & - & 47 & 75 & 0.25 \\
$2009-03-24$ & Clouds & 1.8 & 62 & 65 & 0.25 \\
$2009-06-27$ & Clear & 1.0 & 44 & 95 & 0.20 \\
$2009-06-28$ & Clear & 1.2 & 70 & 90 & 0.20 \\
$2009-10-17$ & Clouds & 1.2 & 79 & 85 & 0.25 \\
$2009-10-18$ & Clouds & 1.5 & 58 & 70 & 0.30 \\
$2009-10-19$ & Clouds & 1.8 & 75 & 75 & 0.20 \\
$2009-10-22$ & Clear & 1.6 & 86 & 90 & 0.20 \\
$2009-10-23$ & Clear & 1.2 & 50 & 95 & 0.15 \\
\hline
\end{tabular}

Notes. For each night, we present average values of the seeing, humidity, transparency of the atmosphere, and the extinction in the $V$-band. (a) Owing to technical problems, there are no data from the seeing monitor for these nights.

grating was used to achieve a nominal spectral resolution of $\sim 10.7 \AA$ (full width at half maximun - FWHM) and a wavelength range of $\lambda \lambda 3620-7056 \AA$. All the targets observed during this run correspond to the first sub-sample described in the previous section. After that date, the V600 grating was used, with a nominal resolution of $\sim 5.4 \AA$ ( FWHM) and a wavelength range of $\lambda \lambda 3845-7014 \AA$. Most of the targets observed during this run correspond to the DMS sub-sample. The main reason for that change was the upgrade of the PMAS CCD, from a $2 \mathrm{k} \times 4 \mathrm{k}$ chip to a $4 \mathrm{k} \times 4 \mathrm{k}$ chip, which nominally increased the wavelength range by a factor of two at the same spectral resolution. This increase was hampered by a vignetting effect that affects about $30 \%$ of the fibres/spectra, at the edges of the CCD (see Fig. 3). Fibers positioning in the entrance slit is performed in such a way that the central and outer regions of the hexagon are located in

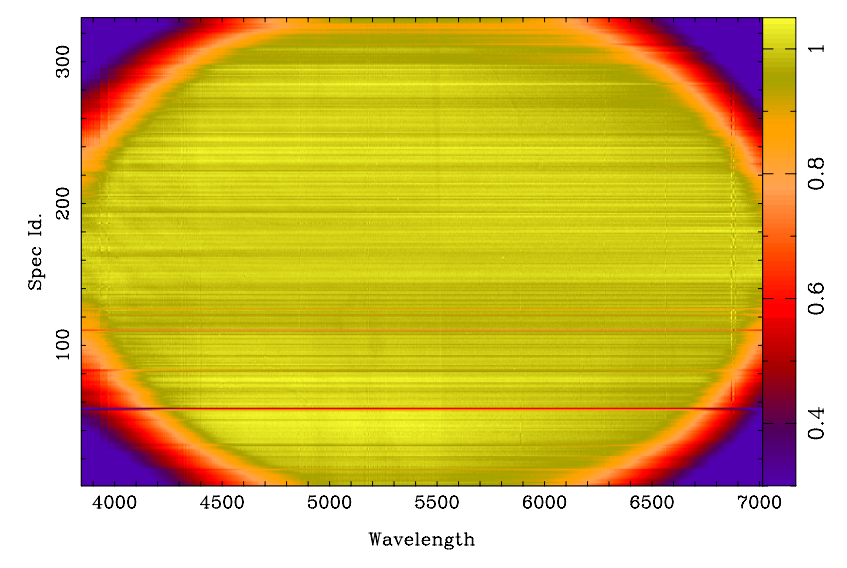

Fig. 3. Intensity map of the differential transmission fibre-to-fibre for the V600 grating. The sharp decline in the transmission at the edges of the image is due to vignetting. About $15 \%$ of the CCD is affected by this effect.

Table 3. PMAS/PPaK instrumental setups.

\begin{tabular}{lcc}
\hline \hline Grid type & V300 & V600 \\
\hline Spectral coverage & $3620-7056 \AA \AA$ & $3845-7014 \AA \AA$ \\
Dispersion & $3.2 \AA / \mathrm{px}$ & $1.5 \AA / \mathrm{px}$ \\
$F W H M^{a}$ & $10.7 \AA$ & $5.4 \AA$ \\
Exposure time $^{b}$ & $600 \mathrm{~s} \times 3$ & $600 \mathrm{~s} \times 3$ \\
\hline
\end{tabular}

Notes. ${ }^{(a)}$ Spectral resolution $(F W H M)$ for PPaK IFU with $150 \mu \mathrm{m}$ fibres. ${ }^{(b)}$ Exposure time for each dithering pointing.

the central regions of the slit, while the outer regions of the slit corresponds to an annular ring. Owing to this positioning of the fibres over the detector for this instrument, the vignetted regions correspond spatially to an annulus ring at about $\sim 15^{\prime \prime}$ from the centre of the IFU. Despite this effect, all the fibres are not affected by vignetting more than $\sim 50 \%$ of their spectral range.

\section{Data reduction}

The large amount of data generated during IFS observations ensures that the establishment of a precise and quick procedure for the data reduction was one of the key points to test during this study. The reduction was performed using a fully automatic pipeline, i.e. one that operates without human intervention, producing both scientifically useful frames and a set of quality control measurements (on a web-based interface). The pipeline was based on the routines included in the R3D (Sánchez 2006) and E3D (Sánchez 2004) packages. The reduction consists of the standard steps for fibre-based integral-field spectroscopy. For the V300 data, a master bias frame was created by averaging all the bias frames observed during the night and was then subtracted from the science frames. In the case of the V600, observed with the new PMAS CCD, which has basically no structure in the bias, a single value bias-level (derived from the the over-scan values), is subtracted from the frames. The different exposures taken at the same position on the sky were then combined, clipping the cosmic rays using IRAF $^{2}$ routines. The next step was to determine the locations of the spectra on the CCD,

${ }^{2}$ IRAF is distributed by the National Optical Astronomy Observatories, which are operated by the Association of Universities for Research in Astronomy, Inc., under cooperative agreement with the National Science Foundation. 
using in this case a continuum-illuminated exposure taken before the science exposures. Each spectrum was then extracted from the science frames.

To reduce the effects of the cross-talk, we adopted a modified version of the Gaussian-suppression proposed by Sánchez (2006), and fully described in Sánchez et al. (2011b). This technique assumes a Gaussian profile for the projection of each fibre spectrum along the cross-dispersion axis, and performs a Gaussian fitting to each of the fibres after subtracting the contribution of the adjacent fibres in an iterative process. The crosstalk is reduced to less than $1 \%$ when adopting this method.

Each spectrum extracted along the dispersion direction is stored in a row-stacked-spectrum file (RSS, Sánchez 2004). Wavelength calibration was performed using HeHgCd lamp exposures obtained before and after each pointing, yielding an accuracy of $\sim 10 \%$ of the nominal pixel scale (i.e., $\sim 0.3 \AA$ for the V300 grating and $\sim 0.15 \AA$ for the V600 one). Differences in the relative fibre-to-fibre transmission throughput were corrected by comparing the wavelength-calibrated RSS science frames with the corresponding frames derived from sky exposures taken during the twilight.

As mentioned before, $\mathrm{PPaK}$ is equipped with 36 fibres to sample the sky, distributed around the science fibre-bundle, in six small bundles of six fibres each one, at a distance of $\sim 75^{\prime \prime}$ from the centre of the FoV (seen Fig. 5 in Kelz et al. 2006). The objects targeted in these studies cover a substantial fraction of the FoV of the central PPaK bundle, but most of the sky fibres are free from emission from the targets. The procedure adopted to derive the night-sky spectrum was to combine the spectra corresponding to these fibres, performing a $2 \sigma$ clipping rejection to remove any possible contamination ${ }^{3}$. Finally, the sky-spectrum was subtracted from all the spectra in the corresponding frame.

Spectrophotometric standard stars from Oke (1990) were observed during the nights to perform the flux calibration. The extracted spectra of these stars were compared with the fluxcalibrated spectra available from the webpage of the observatory ${ }^{4}$, obtaining the transformation function from observed counts to intensity. In this step, the routines in the R3D package take into account the airmass and extinction of the observation of both the spectrophotometric standard stars and the science targets. This procedure ensures a good relative flux calibration from the blue to the red part of the spectra, if the weather conditions along the night were stable. However, an absolute offset between the derived and real fluxes are expected because of the incomplete spatial coverage of the FoV in the observations of the calibration star despite the large fibre size of PPaK (2.7"), and second order effects such as small inaccuracies in the centring of the star and the effects of the variation in the seeing.

After reducing the frames corresponding to each particular pointing, the science spectra corresponding to the three dithered exposures were combined to form a single frame of 993 spectra. To take into account possible variations in the atmosphere transmission during the exposures, the procedure rescales the spectra to a common intensity by comparing the integrated spectra within an aperture of 20"1diameter, chosen as a compromise between the signal-to-noise ratio $(\mathrm{S} / \mathrm{N})$ and the depth of the images, and to avoid seeing effects. The final position tables for the individual fibres are generated by taking into account their relative

\footnotetext{
3 We adopted this criterion since a $3 \sigma$ clipping rejection does not remove the contamination by stars in the field, external regions of the galaxies, or a companion galaxy in IFU observations, owing to the positions of the fibres.

${ }^{4}$ http://www. caha.es/pedraz/SSS/Oke/oke.html
}

positions from the PPaK central bundle and the offsets provided for the dither scheme. The information included in the combined spectra and position tables are used to create the final datacube.

The next step in the reduction process is to perform the differential atmospheric refraction (DAR) correction. To perform this correction, the pipeline creates a $2 \mathrm{D}$ image by combining the flux within 4500-5500 $\AA$ (approximately the $V$-band), and searches the peak emission within a region between $[15: 62$, 15:62] pixels. This centroid is used as initial reference, that will look for any shift in this centroid along the wavelength within a box of $6 \times 6$ pixels. Once the shift is determined, a polynomial order fitting of order three is applied to smooth the correction, and the shift is applied wavelength to wavelength, re-centring the cube, and correcting for the DAR.

To get the highest possible quality of absolute flux calibration, we re-calibrated our data using SDSS photometry, whenever available (37 targets, 33 of them of high enough quality for the analysis). Of the five SDSS filters (ugriz), our spectrum covers the passbands of two, namely the $g\left(\lambda_{\text {eff }}=4770 \AA\right)$ and $r$ $\left(\lambda_{\text {eff }}=6231 \AA\right)$ filters. We measured the counts of each galaxy in these SDSS images inside a $30^{\prime \prime}$ diameter aperture. These counts were converted to flux following the counts-to-magnitude prescription in SDSS documentation ${ }^{5}$. We cross-checked this method by measuring the magnitudes of stars on each field and comparing them with SDSS DR6 photometric catalogue, obtaining a magnitude dispersion of less than 0.05 for $g<17.5$ mag.

We then extracted the spectrophotometry from our reduced cubes, summing the flux of individual spectra inside a $30^{\prime \prime}$ diameter aperture around the peak intensity of the object, and convolving this spectrum with the SDSS $g$ and $r$ filter passbands (Jester et al. 2005). Using these two data pairs, a scaling solution of the form

$F_{\text {corr }}(\lambda)=F_{0}(\lambda) \times a$

was derived for each band, where $F_{\text {corr }}(\lambda)$ and $F_{0}(\lambda)$ are the re-calibrated and original fluxes, respectively, and $a$ is the calibration constant. The root-mean-square (rms) of these values is $\sim 4 \%$ in the $g$ band and $\sim 5 \%$. Finally, the average of the two scaling constants derived for the $g$ and $r$ filters is adopted. In this way, we were able to recalibrate the spectrum to match the SDSS photometry.

Figure 4 shows two examples of the resulting re-calibrated spectra observed with the V300 and V600 grating. In addition to the values for the $g$ and $r$ filters used in this re-calibration, we present the derived values for SDSS $u$ and $i$ bands. The figure also includes the fluxes corresponding to the Johnson $B, V$ and Cousin $R_{\mathrm{C}}$ magnitudes determined on the basis of the SDSS photometry and the transformation equations presented by Jester et al. $(2005)^{6}$. There is good agreement in all cases suggesting that this re-calibration method is reliable.

Figure 5 (top panels) shows the $g$ and $r$-band magnitudes derived from the datacubes and the SDSS images before (blue solid circles) and after (red solid squares) the recalibration process. Both sets of photometric data match for $85 \%$ of the galaxies within a range of \pm 0.15 mag before the recalibration (just four objects have a larger dispersion below $\pm 0.25 \mathrm{mag}$ ). The possible differences in colours is explored in Fig. 5, bottom panel. Any possible error in the relative flux calibration from blue to red would produce a change in colour. The dispersion (rms)

\footnotetext{
5 http://www.sdss.org/dr7/algorithms/fluxcal.html\# counts 2 mag

6 Summary of the transformations in http://www.sdss.org/dr7/ algorithms/sdssUBVRITransform.html
} 

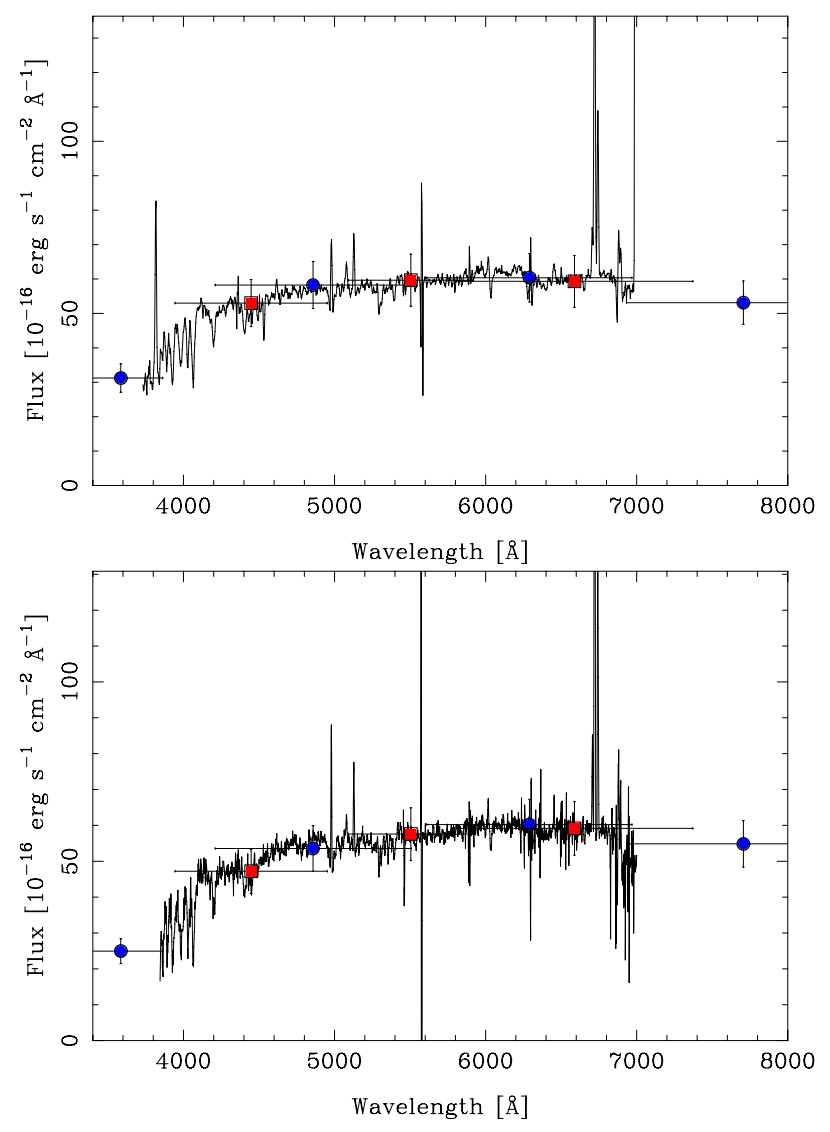

Fig. 4. Examples of the flux re-calibration based on SDSS photometry for CGC071-96 (observed with the V300 grating, top panel) and CGCG430-046 (V600 grating, bottom panel). The values for the SDSS $g$ and $r$ filters used in the re-calibration are presented with blue solid circles, as well as the derived values for SDSS $u$ and $i$ filters. Johnson $B, V$, and Cousin $R_{\mathrm{C}}$ magnitudes are also shown (red solid squares) to test the flux calibration.

around the one-to-one relation is $\sim 0.1 \mathrm{mag}$, consistent with the expected errors from propagating both the SDSS photometric errors $(\sim 4-5 \%)$ and the absolute flux calibration accuracy of our data $(\sim 8 \%)$.

In a few cases (11 targets) when the SDSS photometry is unavailable for a galaxy, we obtained broad-band images using CAFOS at the $2.2 \mathrm{~m}$ telescope of the Calar Alto Observatory, Spain. The observations were carried out for six nights between January 28th and March 30th, 2010, when images with the Johnson $B, V, I$, and Cousin $R_{\mathrm{C}}$ filters were obtained. The CCD camera was equipped with a SITe pixel chip. The pixel size was $24 \mu \mathrm{m}$, corresponding to $0.53^{\prime \prime}$. Sky flats were exposed in every dawn and dusk phase. Bias subtraction, flat-field division, and trimming were performed in the usual manner, using the IRAF package.

We photometrically calibrated these images using GSC2.3 (Lasker et al. 2008) and USNO-B (Monet et al. 2003) catalogues as follows. We obtained $B$ and $V$ photometry for our galaxies using the qphot routine of the IRAF and adjusted the zero-point (ZP) magnitude to the GSC2.3 and USNO-B photometry. For these $\mathrm{ZP}$ calibrations, we measured the magnitudes of several stars (7-8) in the same field of our galaxies and we then determined the difference between our magnitudes and the catalogued ones. In general, the dispersion obtained for the ZP determined using the USNO-B catalogue was nearly 0.4 mag on average, so we decided to use the GSC2.3 ZP with a dispersion
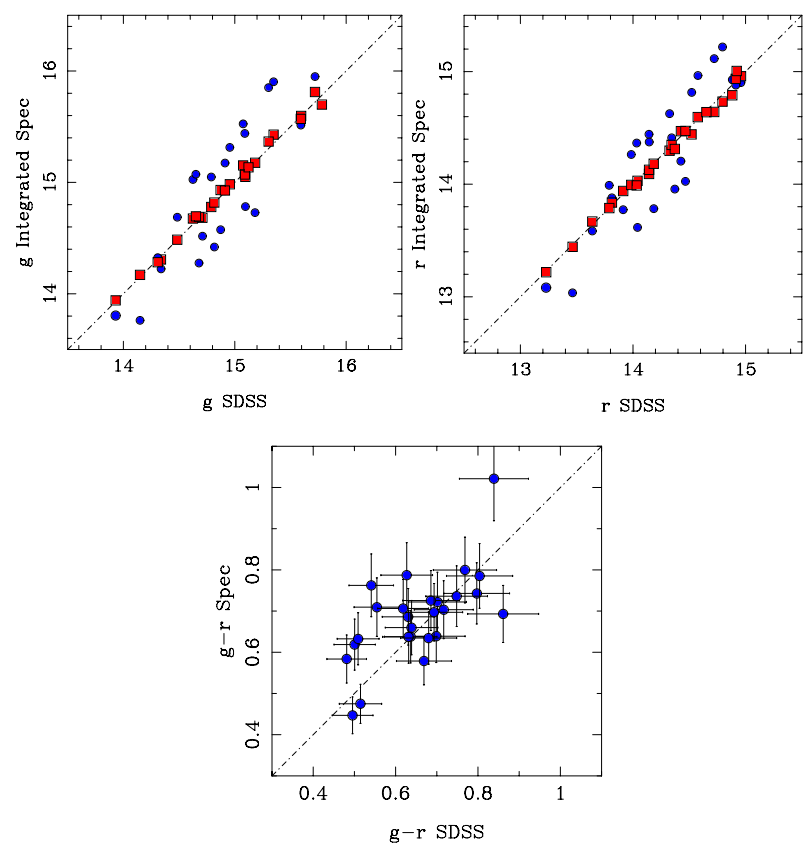

Fig. 5. Top: comparison of $g$ (right panel) and $r$-band (left panel) magnitudes derived from the integrated spectrum within an aperture of $30^{\prime \prime} /$ diameter extracted from the V600 datacubes and the corresponding magnitudes derived from the SDSS images, before (blue solid circles) and after (red solid squares) the flux calibration described in the text. Bottom: comparison of $g-r$ colour. Considering the errors in the absolute flux calibration ( $\sim \%$ ) and between $g$ and $r$ band (around 4 $5 \%$ ), the expected errors in the colours are $\sim 0.1 \mathrm{mag}$, consistent with the errors in SDSS photometric data.

of 0.2 mag on average. One ZP was calculated for each image with this method. Following the same procedure described before, we then convolved a similar aperture spectra extracted from the datacubes with the $B$ and $V$ filter responses, obtaining another pair of data sets for recalibrating these cubes. Since the stars from the GSC2.3 catalogue used in this work presented errors of $0.1-0.4 \mathrm{mag}$, we expect our recalibration procedure to ensure an absolute spectrophotometric calibration of better than a $\sim 15 \%$ in all the spectral range.

Finally, the pipeline performs a set of quality control measurements that are accessible to the user on a web-based interface, as indicated above. Among these measurements, the most relevant are (i) cross-checks of the focus accuracy of the instrument; (ii) independent estimates of the accuracy of the wavelength calibration (by comparing the nominal wavelength of the most prominent emission lines included in the sampled wavelength range with the measured ones on the data, for each individual spectra within the reduced RSS); (iii) estimates of accuracy of the sky-subtraction (from the residual flux of the strongest and most variable night-sky emission line in the considered wavelength range - OI $5577 \AA$ ); (iv) estimate of the night-sky brightness at the moment of observation; (v) estimate of the depth of the final datacubes (by computing the $\mathrm{S} / \mathrm{N}$ in the spectral range 5670-5730 $\AA \AA$ ). For the observations presented in this paper, the average $5 \sigma$ detection limit is $\sim 22.5-23.0 \mathrm{mag} / \operatorname{arcsec}^{2}$ ( $V$-band) and the accuracy of the wavelength calibration is $\sim 0.3 \AA$. In Table 4 , we present these values, together with the $V$-band magnitude of the sky, for each observing night. On the basis of these quality control procedures, it is possible to discard seven datacubes owing to their poor quality. Most of these objects were observed under non-photometric 
Table 4. Automatic quality control measurements made by the pipeline.

\begin{tabular}{lccc}
\hline \hline $\begin{array}{l}\text { Observing } \\
\text { date }\end{array}$ & $\begin{array}{c}5 \sigma \text { limit } \\
V \text {-band } \\
(\mathrm{mag})\end{array}$ & $\begin{array}{c}\text { Sky } \\
V \text {-band } \\
\text { magnitude }\end{array}$ & $\begin{array}{c}\text { Wavelength } \\
\text { calibration } \\
(\AA)\end{array}$ \\
\hline $2007-06-22$ & 22.73 & 20.50 & 0.30 \\
$2007-06-23$ & 22.50 & 20.50 & 0.30 \\
$2009-03-19$ & 23.02 & 20.25 & 0.43 \\
$2009-03-20$ & 23.02 & 20.28 & 0.34 \\
$2009-03-21$ & 22.92 & 20.10 & 0.26 \\
$2009-03-22$ & 22.84 & 20.12 & 0.39 \\
$2009-03-23$ & 21.54 & 19.07 & 0.22 \\
$2009-03-24$ & 23.19 & 19.01 & 0.32 \\
$2009-06-27$ & 23.05 & 20.13 & 0.35 \\
$2009-06-28$ & 23.75 & 18.35 & 0.30 \\
$2009-10-17$ & 22.55 & 20.05 & 0.15 \\
$2009-10-18$ & 21.58 & 19.42 & 0.20 \\
$2009-10-19$ & 22.00 & 19.83 & 0.15 \\
$2009-10-22$ & 22.42 & 20.13 & 0.15 \\
$2009-10-23$ & 22.32 & 19.73 & 0.23 \\
\hline
\end{tabular}

Notes. The average $5 \sigma$ limit $V$-band magnitude, the $V$-band magnitude of the sky, and the accuracy of the wavelength calibration are presented for each night.

conditions, and during the control process were clearly identified as ones to be discarded, as expected.

\section{Analysis and results}

To extract the physical properties of the galaxy from the data set, it is necessary to identify the emission lines produced by ionised gas in the galaxies, and decouple this emission from the underlying stellar population. Particular care has to be taken in this decoupling technique, because some of the emission lines (e.g. $\mathrm{H} \beta$ ) may be strongly affected by underlying absorption features. Several authors have developed different fitting codes to obtain information about the stellar content and star formation history of galaxies (e.g., Cid Fernandes et al. 2005; Ocvirk et al. 2006; Sarzi et al. 2006; Koleva et al. 2009; MacArthur et al. 2009). In this paper, we adopted the decoupling method proposed by Sánchez et al. (2011b), based on a full spectrum modelling. This method involves a linear combination of multiple stellar populations and the non-linear effects of dust attenuation to obtain information about the ionised gas and the stellar content of the galaxies, and can be applied to each of the individual spectra of the datacubes to obtain the $2 \mathrm{D}$ distribution of the parameters derived with this procedure.

In this section, we present the method (Sect. 5.1) and analysis of the accuracy of the physical properties derived with this method (Sect. 5.2). We present in Sect. 5.3 a comparison with the SDSS data, and explore differences in the properties derived from two different apertures obtained from the data in Sect. 5.4. Finally, a first analysis from the 2D spectroscopic data is presented (Sect. 5.5).

\subsection{Decoupling the stellar population and gas content}

The method for the decoupling of the stellar populations from the emission lines can be summarised as follows (for a more detailed description, see Sánchez et al. 2011b): (i) a set of detected emission lines was identified in the spectrum; (ii) the underlying stellar population was fitted by a linear combination of a simple grid of six single-stellar populations (SSPs) templates by Vazdekis et al. (2010), with three ages $(0.089,1.000$,
CGC071-096

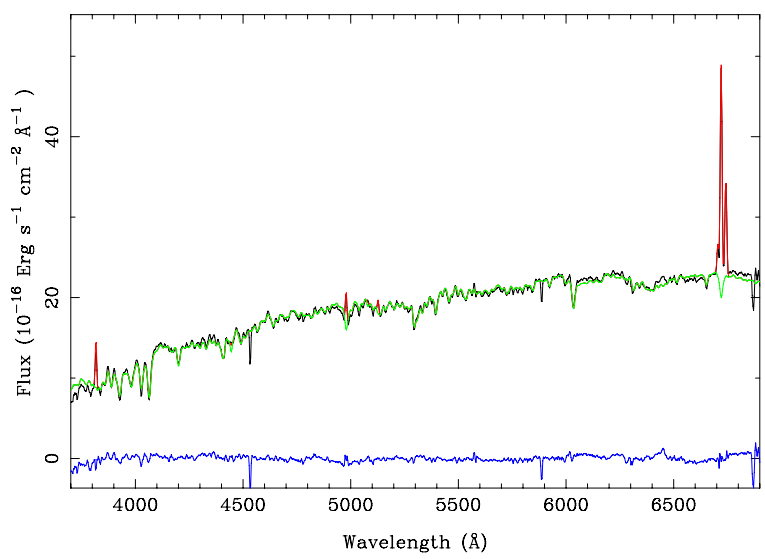

Fig. 6. Example of the decoupling of emission lines and underlying stellar population for CGC071-096. We show the central spectrum as a black line, the best-fit model of the emission lines in red, and the best-fit stellar population, modelled as a linear combination of different SSPs, plus the computed dust extinction, in green. Finally, the differences between the real spectrum and the final fitting is shown in blue.

and $17.7828 \mathrm{Gyr})$ and two metallicites $(Z=0.0004 \text { and } 0.03)^{7}$. These templates were first corrected for the appropriate systemic velocity and velocity dispersion (including the instrumental dispersion), taking into account the dust attenuation ${ }^{8}$. In addition, a spectral region of $30 \AA$ width around each detected emission line was masked prior to the linear fitting, including also the regions around bright sky-lines (Sánchez et al. 2007a); (iii) once we derived a first approximation of the spectrum of the underlying stellar population, this was subtracted from the original spectrum to obtain a pure emission-line spectrum; (iv) to derive the intensity of each detected emission line, this emission-line spectrum was fitted to a single Gaussian function per emission line plus a low order polynomial function for the local continuum; (v) a pure gas-emission spectrum was created, based on the results of the last fitting procedure, using only the combination of Gaussian functions. The pure gas-emission model was then subtracted from the original spectrum to produce a spectrum of gas-free spectra; (vi) this spectrum is fitted again by a combination of SSPs, as described before (but without masking the spectral range around the emission lines, in this case), deriving the luminosity-weighted age, metallicity and dust content of the composite stellar population; (vii) finally, individual emission-line fluxes are measured in each spectrum by considering spectral window regions around the most prominent emission lines (fitting of Gaussian funtions to [O II] $\lambda 3727, \mathrm{H} \beta$, [O III] $\lambda \lambda 4959,5007, \mathrm{H} \alpha,[\mathrm{N}$ II] $\lambda 6548,83,[\mathrm{~S}$ II] $\lambda \lambda 6717,6731)$. With this method, we obtain information about the ionised gas and the stellar content in the analysed spectrum.

This decoupling method can be applied to any galaxy spectrum, either integrated spectrum or individual spectra in the datacubes, to analyse the spatially resolved spectroscopic properties. As an example, we show in Fig. 6 the best fitted gasemission (red line) and stellar population (green line) spectra obtained when applying this decoupling method on the central spectrum of CGC071-096 galaxy (black line). The differences

\footnotetext{
7 This grid of templates has been also adopted by Viironen et al. (2011) in their analysis of the UGC9837 galaxy from this data set.

8 The dust attenuation is also a free parameter in this decoupling method. The extinction law of Cardelli et al. (1989) is adopted, with a ratio of total to selective attenuation $R_{V}=3.1$ (Jenkins 1987).
} 
between the real spectrum and the final fitting are shown with a blue line. These residuals represent an error of $\sim 7 \%$ in the signal of the global spectrum, and illustrate the close agreement of the global fitting.

\subsubsection{Star formation history}

To explore a more realistic interpretation of the computed stellar population, we decided to compare the galaxy data not only with SSPs, but also with spectra obtained from a given star formation history (SFH). With this aim, we used the population synthesis models of Vazdekis et al. (2010). These models provide the time $(T)$ evolution of the synthesised spectra of galaxies characterised by an initial metallicity $(Z)$, a star formation history (SFH), and an initial mass function (IMF) $\left(F_{\lambda}(T)[Z, S F H, I M F]\right)$. We considered a pure exponentially declining SFH (Sandage 1986):

$\operatorname{SFR}(t)=\exp \left(-\left(T_{0}-t\right) / \tau\right)$

where the steepness of the decay are regulated by a single parameter $\tau$. To obtain the final spectra, we considered a simple (not delayed) exponential, where $T_{0}=15.5 \mathrm{Gyr}$ is the age of the galaxy today, and a Salpeter IMF (a unimodal IMF with $\mu=1.3$ ). With these input data and using the tools provided in the MILES (Medium-resolution Isaac Newton Telescope library of empirical spectra) website ${ }^{9}$, we created a grid of spectra with solar and sub-solar metallicities $(Z=0.019,0.0004)$ and $\tau$ from 1 to 19 in steps of 2 Gyr, with a $\mathrm{S} / \mathrm{N} \sim 50$ per pixel in the continuum, and spectral resolutions corresponding to the V300 and V600 grism.

To relate the characteristic scale time parameter $\tau$ to its corresponding stellar population parameters, i.e., luminosityweighted age and metallicity, we applied the fitting procedure explained in the previous section, using as input the spectra generated from these SFHs. In Fig. 7, we represent the luminosityweighted age and metallicity derived for the grid of spectra from the different SFH parametrised by $\tau$ as a function of this parameter. As can be seen, we obtain a clear dichotomy for the metallicity. This is expected because we consider two different metallicities for the spectra created from a given SFH. In this sense, the metallicity is well recovered for all the values of $\tau$, although the metallicity is over-estimated in the case of the solar metallicity owing mainly to the limited available values in the grid of models using in this experiment.

We obtain old stellar ages for low values of $\tau$ and younger ages until $\tau>10$, for which the age is almost constant. This agrees with Gavazzi et al. (2002), who found that dwarf and normal elliptical galaxies, i.e. galaxies with older stellar populations, are well characterised by $\tau \leq 3$. They also found that galaxies with younger stellar populations have $\tau \geq 4$, such as spiral galaxies ( $\mathrm{Sa}-\mathrm{Sb}$ ) with values of $4<\tau<6$, and blue compact dwarfs with $\tau \geq 7$. The equivalences obtained here (Fig. 7) will be used to characterise the stellar populations in Sect. 5.2.4.

\subsection{Accuracy of the derived properties}

Following the idea of recent works based on IFU observations, (e.g., Bershady et al. 2010b; Sánchez et al. 2011b), we now explore the accuracy of the measurements of different parameters of our interest (e.g., emission-line fluxes, redshift, velocity dispersion, dust extinction, average stellar population parameters).

\footnotetext{
9 http://www.iac.es/proyecto/miles/pages/webtools/ get-spectra-for-a-sfh.php
}
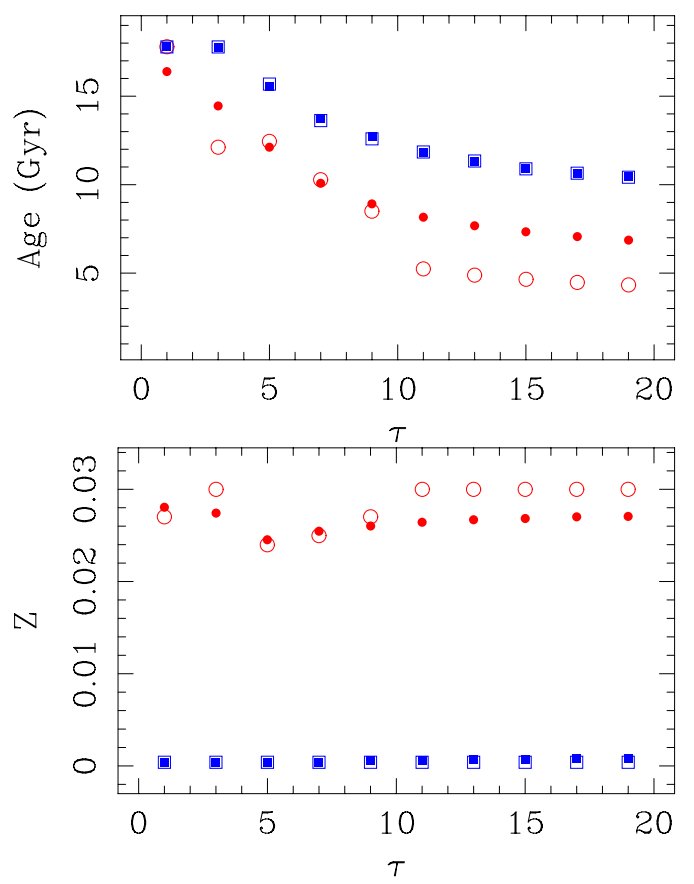

Fig. 7. Corresponding sets of the stellar population parameters, i.e. luminosity-weighted age (top panel) and luminosity-weighted metallicity (bottom panel), and characteristic scale time parameter $\tau$ for the two SFH considered (red circle for solar metallicity, blue squares for subsolar). We present the results of the fitting procedure applied to mock galaxies with the V600 resolution, when the dust attenuation is fixed (filled symbols) and computed (open symbols).

We created a set of mock spectra of galaxies with different kinematic galactic parameters (velocity dispersion, redshift), underlying stellar populations (old or young SSP), ionised gas, and $\mathrm{S} / \mathrm{N}$ in the continuum. These mock spectra were generated as follows. First, we considered an underlying stellar population using the stellar population models provided by Vazdekis et al. $(2010)^{10}$. We studied four possibilities for the underlying stellar population: a single stellar population (young -0.7 Gyr and solar metallicity $(Z=0.019)$ - or old -12.6 Gyr and metallicity of $Z=0.004)$, or two stellar populations mixed in different ratios ( $10 \%$ of young plus $90 \%$ of old stellar population - 2SSP$10-$, and $1 \%$ of young plus $99 \%$ of old stellar population 2SSP-01). Next, we added an emission-line spectrum to simulate the ionised gas in the mock galaxy. To obtain more realistic data, we measured the emission lines in several regions of NGC628 from the 2D spectroscopic data presented by Sánchez et al. (2011b), and scaled these values to obtain similar ones to those measured in a real galaxy of our sample (see e.g. Viironen et al. 2011). The nebular continuum is considered negligible. We then applied different values of dust attenuation, identically for the stellar continuum and the emission lines. We then considered the kinematics of the mock galaxy, i.e., we broadened the spectrum to a given velocity dispersion $\sigma$ and shifted it to a given redshift $z$. Finally, we added noise to the spectrum to simulate a real galaxy observation. As result of this process, we obtained a spectrum of a mock galaxy with typical properties that can be altered to help us determine the accuracy of our measurements.

In the next subsections, we present the different studies that we carried out to analyse the accuracy of the measurements of the dust attenuation (Sect. 5.2.1), kinematics (Sect. 5.2.2),

10 http://www.iac.es/proyecto/miles/ 

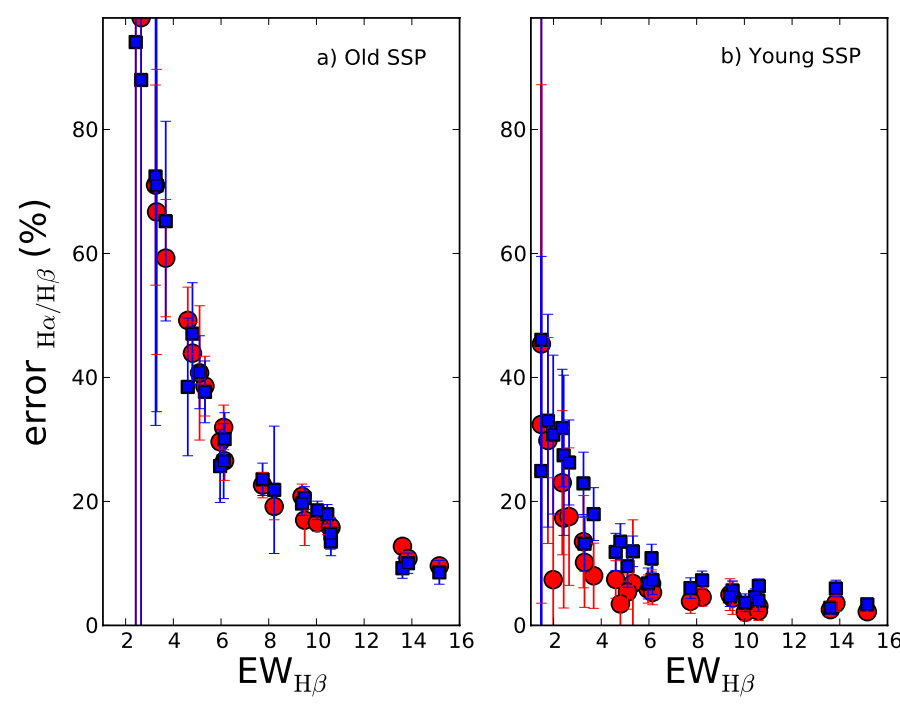

Fig. 8. Relative errors in the $\mathrm{H} \alpha / \mathrm{H} \beta$ ratio when the dust attenuation is fitted (blue squares) or fixed (red circle) for different equivalent widths of the $\mathrm{H} \beta$ emission line $\left(E W_{\mathrm{H} \beta}\right)$, for a spectrum of a mock galaxy with a pure old (left panel) or young underlying stellar population (as explained in the text).

ionised gas (Sect. 5.2.3), and underlying stellar population (Sect. 5.2.4), of the galaxies observed with the two setups (V300 and V600) used in this work.

\subsubsection{Dust attenuation}

The treatment of the dust attenuation may affect the resulting derived parameters of the stellar population (i.e., the luminosityweighted age and metallicity, Calzetti 2001; Muñoz-Mateos et al. 2007, 2009). As part of the decoupling method explained above, we adopted the Cardelli et al. (1989) law, which may not be the optimal solution for studying the dust attenuation in starforming galaxies (e.g., Calzetti 2001). MacArthur et al. (2009) considered the attenuation law based on the two component dust models of Charlot \& Fall (2000), especially developed for starformation galaxies. Despite the conceptual differences between these two attenuation laws, they have a very similar shapes in the wavelength range of our data, and it is not wide enough to distinguish between these different laws.

To explore the impact of fitting the dust attenuation on the derived parameters, we generated a set of mock spectra of galaxies following the procedure explained above. Since the values of equivalent widths of the $\mathrm{H} \beta$ emission line $E W_{\mathrm{H} \beta}>3.5 \AA$ are suitable for performing an abundance analysis of galaxies despite their dependence on other physical properties of the ionising cluster (e.g., its age), we decided to explore a range of equivalent widths of $E W_{\mathrm{H} \beta} \sim 1.5-15 \AA$ for our mock galaxies. We then applied the fitting technique (i) fixing the value for the dust attenuation, or (ii) computing $A_{V}$ with the fitting program, with values of $A_{V}$ varying between $A_{V}=0.1$ and 1.2 , in steps of 0.1 .

We present the results of this experiment in Fig. 8, where we show the relative random errors obtained for the $\mathrm{H} \alpha / \mathrm{H} \beta$ ratio (in absolute values) when the dust attenuation was fixed (red circles) and fitted (blue squares), as a function of $E W_{\mathrm{H} \beta}$. In all cases, the $\mathrm{H} \alpha / \mathrm{H} \beta$ ratio is recovered with a relative error $<20 \%$ for $E W_{\mathrm{H} \beta}>8 \AA$ for an old underlying stellar population (left panel), while the relative errors for a young stellar population was $<20 \%$ for $E W_{\mathrm{H} \beta}>3.5 \AA$ (right panel). Although there are no differences in the determination of $\mathrm{H} \alpha / \mathrm{H} \beta$ for an old population depending on the method (fitting or not the dust attenuation), slightly higher errors are found when the dust attenuation is fitted in the case of a young underlying stellar population (right panel in Fig. 8). This is due to the dust attenuation, which is more significant at bluer wavelengths where stronger Balmer absorption lines are found, especially for young stellar populations. In this case, slight variations in the dust attenuation determination can produce differences in the $\mathrm{H} \alpha / \mathrm{H} \beta$ ratio, which are more evident for small equivalent widths of $\mathrm{H} \beta$. We see in Fig. 8 that, in general, the relative errors in the determination of the $\mathrm{H} \alpha / \mathrm{H} \beta$ ratio are similar regardless of the dust attenuation fitting, and the accuracy of the measurement of the gas content is independent of the procedure applied.

\subsubsection{Kinematics}

One of the advantages of using IFU data is that it is possible to analyse the spatially resolved properties of the galaxies. Kinematic studies, in particular, can help us to identify substructures in galaxies such as kinematically decoupled cores in the stellar velocity field and twists in the gas velocity field, in addition to more obscured substructures such as disks and bars. The instrumental setups used in these observations, although limited in terms of spectral resolution, allow some studies of this kind. Data obtained with the V300 and V600 grisms allow the measurement of redshifts, while the resolution of the V600 grism $(F W H M=5.4 \AA)$ will allow measurements of velocity dispersion above $\sim 140 \mathrm{~km} \mathrm{~s}^{-1}$. To assess the accuracy of the measurements of redshift and velocity dispersion for these observing setups, we applied the fitting method explained above to a set of mock spectra from single stellar populations (an old and a young SSP), and $\mathrm{S} / \mathrm{N}=50$ (per pix) in the continuum. In this case, we considered our mock spectra of galaxies at different redshifts (from 0.015 to 0.025 , in steps of 0.001 ) and broadened these to different velocity dispersions (from 150 to $350 \mathrm{~km} \mathrm{~s}^{-1}$, in steps of $25 \mathrm{~km} \mathrm{~s}^{-1}$ ). We applied the decoupling method in the spectral range $\lambda \lambda 3950-4600 \AA \AA$, which comprises clear absorption features suited to this study. In addition, owing to the short wavelength considered in this experiment, a fixed value for the dust attenuation was considered, and not fitted in this case. Finally, for an estimation of the random error in these measurements, we simulated a grid of spectra of each mock galaxy as follows. The average rms of the mock galaxy spectra in areas free of sky lines and galactic emission or absorption lines was calculated and added by applying the bootstrapping method to the SSP+gas model spectrum. This was repeated 100 times, and the fitting was then done on the resulting spectra. The results are presented in Fig. 9, where we show the median relative errors obtained for the velocity dispersion and the redshift of each mock galaxy. The error bars represent the standard deviation computed from the simulations. We find that for both underlying stellar populations, we recover the redshifts with errors $<5 \%$. As expected, the relative errors in determining the velocity dispersion are large for values close to the instrumental setups. It is possible to measure a velocity dispersion with a relative error of $\sim 30 \%$ for $\sigma>200 \mathrm{~km} \mathrm{~s}^{-1}$ (i.e., intrinsic values of the velocity dispersion of galaxies above $140 \mathrm{~km} \mathrm{~s}^{-1}$ ).

\subsubsection{Ionised gas}

Integral field spectroscopy allows the study of the resolved physical properties of the ionised gas in galaxies, such as (i) local 

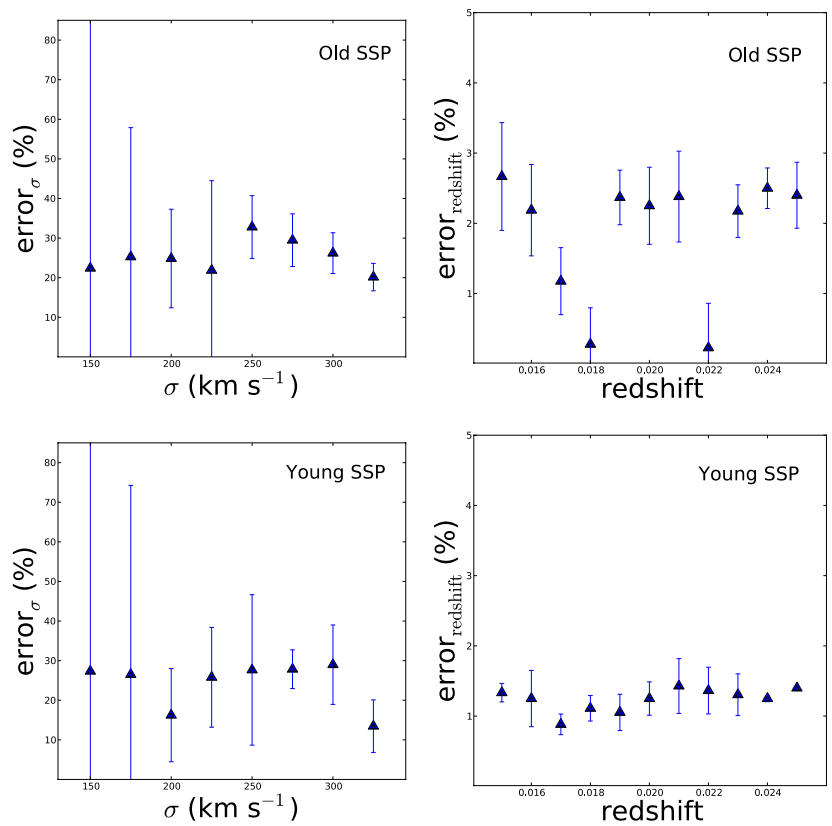

Fig. 9. Estimated relative random errors in the measurements of velocity dispersion (left panels) and redshift (right panels) for mock galaxies with an old (upper panels) and young (lower panels) underlying single stellar populations, as explained in the text, for observations with the medium resolution grism (V600).

nebular reddening estimates based on the Balmer decrement; (ii) the oxygen abundance distributions based on a suite of strong line diagnostics incorporating reddening-corrected line ratios (e.g., $\mathrm{H} \alpha, \mathrm{H} \beta,[\mathrm{O}$ II], [O III], [N II], and [S II]); and (iii) measurements of ionisation structure in $\mathrm{H}_{\text {II }}$ regions and diffuse ionised gas using the well-known and most updated forbidden-line diagnostics in the oxygen and nitrogen lines. For all these studies, it is necessary to measure the fluxes of the emission lines in the individual spectra. To test the accuracy of the measured fluxes for the ionised gas, we followed the procedure explained above to create a set of mock galaxies at redshift $z=0.02$ with a velocity dispersion $\sigma=150 \mathrm{~km} \mathrm{~s}^{-1}$ and $\mathrm{S} / \mathrm{N}=50$ (per pix) in the continuum. We measured the equivalent widths of the main emission lines (see Sect. 5.1) in 29 different regions in NGC628, and created 29 spectra with gaussians corresponding to these widths. In this way, the emission-line ratios of the mock galaxies are realistic and known in advance. We then applied the decoupling method and measured the emission-line fluxes. Finally, we compared these results with the input data from NGC628. In Fig. 10, we plot the relative random error in the $\mathrm{H} \alpha / \mathrm{H} \beta$ ratio as a function of the equivalent width for the $\mathrm{H} \beta$ emission line. We obtain relative errors of the ratio of $\mathrm{H} \alpha / \mathrm{H} \beta<10 \%$ when $E W_{\mathrm{H} \beta}>5 \AA$ for old stellar populations, while we obtain errors of $<20 \%$ when $E W_{\mathrm{H} \beta}>6 \AA$ for young stellar populations. There is a tendency to overestimate the emission of $\mathrm{H} \beta$ for old stellar populations (left panels), while we find the opposite behaviour for young stellar populations (right panels). This is expected because the Balmer absorption lines are deeper for a young stellar population and an accurate decoupling is more difficult if the emission lines are not very intense. In addition, this effect is clearer when the dust attenuation is also fitted in the decoupling method (lower panel in Fig. 10).

\subsubsection{Stellar populations}

As explained before, the decoupling method also provides the best combination of stellar populations to reproduce the
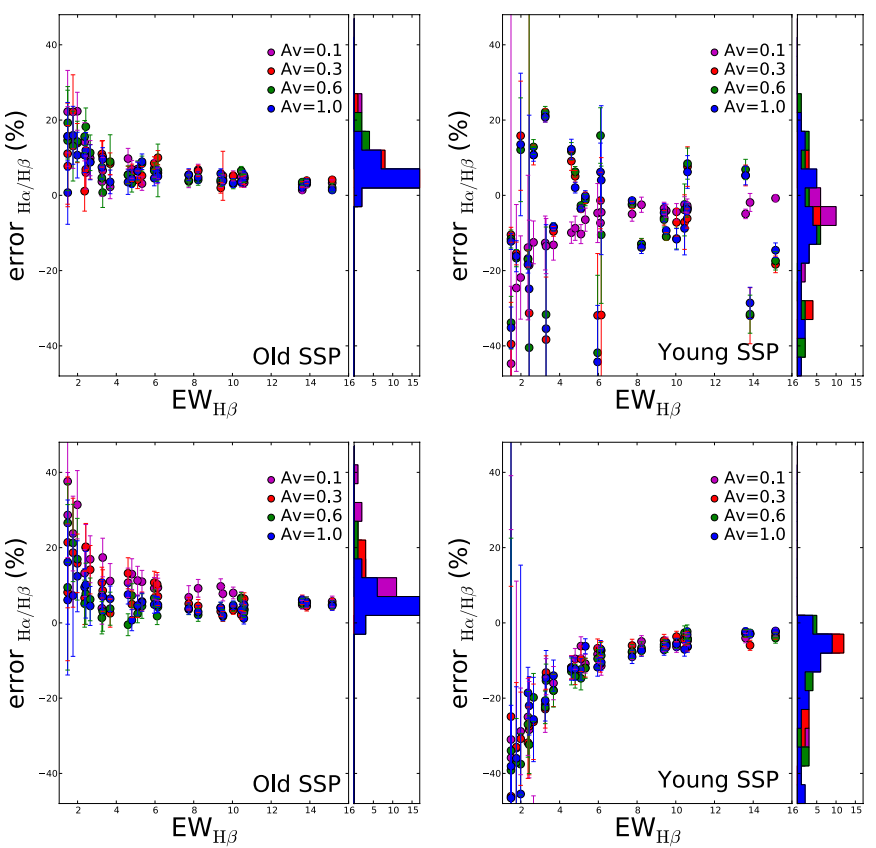

Fig. 10. Study of the recovery of the properties of the ionised gas for observations with the medium resolution grism (V600). Estimated relative random errors in the measurements of the $\mathrm{H} \alpha / \mathrm{H} \beta$ ratio as a function of the equivalent width of $\mathrm{H} \beta$ emission lines $\left(E W_{\mathrm{H} \beta}\right)$ for mock galaxies with an old (left panels) and a young (right panels) underlying stellar population and different values for the dust attenuation: magenta for $A_{V}=0.1$, red for $A_{V}=0.3$, green for $A_{V}=0.6$, and blue for $A_{V}=1.0$. Top panels are for dust attenuation fixed in the program, and bottom panels for dust attenuation fitted in the program. Each block of the histogram represents an error of $5 \%$.

underlying stellar population of galaxies. In this section, we study the recovery of the luminosity-weighted age and metallicity from the data obtained in this work. We applied the decoupling method to spectra of mock galaxies with different underlying stellar populations and gas content (see Sect. 5.2), at redshift $z=0.02$ with a velocity dispersion $\sigma=150 \mathrm{~km} \mathrm{~s}^{-1}$. We considered different $\mathrm{S} / \mathrm{N}(=5,10,25,50,80,100$ per pixel $)$ in the continuum of the spectra. In addition, we created a grid of spectra from each mock galaxies for error analysis, following the procedure described in previous sections. We then applied the decoupling method in two cases: (i) the dust attenuation is a well known and fixed value (results for the luminosity-weighted age in Fig. 11), and (ii) the dust attenuation is a free parameter in the fitting procedure procedure (results in Fig. 12). In both cases, we represent the relative error in the luminosity-weighted age for the four cases of underlying stellar population. The error bars show the standard deviation for these values computed from 100 simulations, as in previous sections.

As can be seen, the relative random errors in the luminosityweighted age for mainly old stellar populations (panels $a, c$ and $d$ in Figs. 11 and 12) are $\sim 30-55 \%$, while the estimated errors and their standard deviation are higher, on average, when considering younger stellar populations (panel $b$ ). In addition, the relative errors are significantly larger when fitting simultaneously the dust attenuation (Fig. 12). It is clear from this analysis that the spectral range (similar for data obtained with V300 and V600 grids) is not wide enough to break the age-metallicity-dust degeneracy. Additional information in other spectral bands, such as the ultraviolet and infrared, are desirable to obtain a reliable independent estimate of the dust attenuation. In this case, dust 

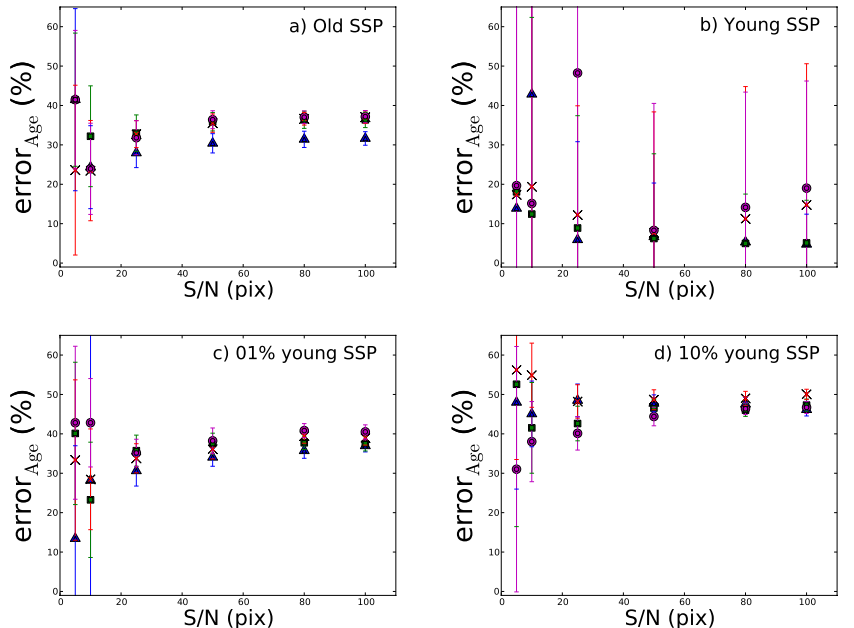

Fig. 11. Study of the recovery of the luminosity-weighted age as a function of the $\mathrm{S} / \mathrm{N}$ in the continuum for the $\mathrm{V} 600$ grid. In this case, the dust attenuation has been assumed to be known and fixed when applying the decoupling method. Different colours are used for different values of the $\mathrm{H} \beta$ emission line.
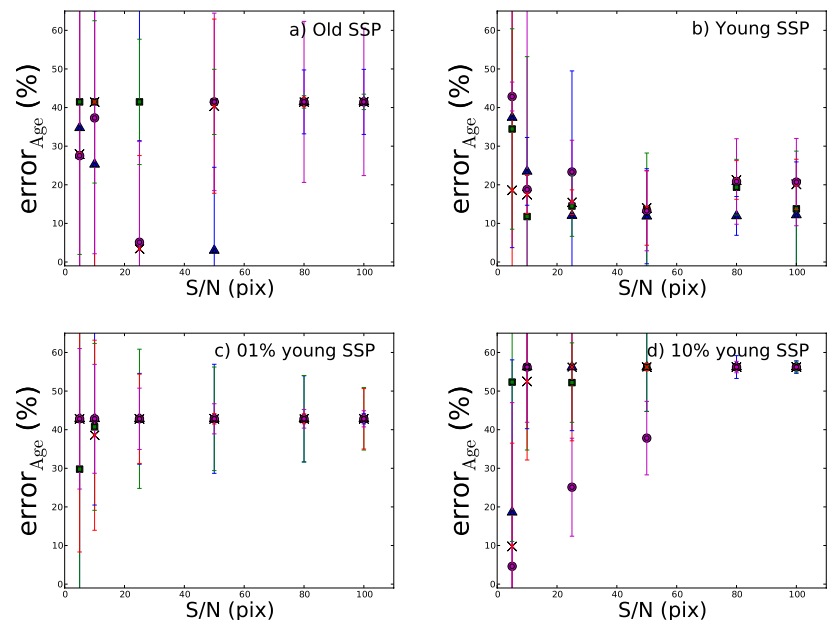

Fig. 12. Same figure as Fig. 11 when the dust attenuation is also computed with the decoupling method. In this case, the estimated random errors (and their standard deviations) are significantly larger than in the previous example.

attenuation would be fixed to this value in the fitting process and a more accurate determination of the remaining of parameters obtained.

In addition, the relative random errors in the luminosityweighted metallicity are extremely large, above $100 \%$ in all cases. This indicates that the grid of templates, based in six stellar population models, are too simple for the estimate of metallicity. As noted by MacArthur et al. (2009) and shown by Sánchez et al. (2011b), the analysis of the stellar populations is limited by the templates used. Tentative tests with a grid of 20 and 25 SSPs decrease the error in the luminosity-weighted metallicity to $\sim 35 \%$, hence a study of the stellar populations is possible with this data by assuming the long computational time necessary. We highlight, in any case, that the recovery of the kinematics and the properties of the ionised gas are well performed with the simple grid considered in this paper, and a further analysis of the stellar population parameters is feasible with an appropriate grid of templates.
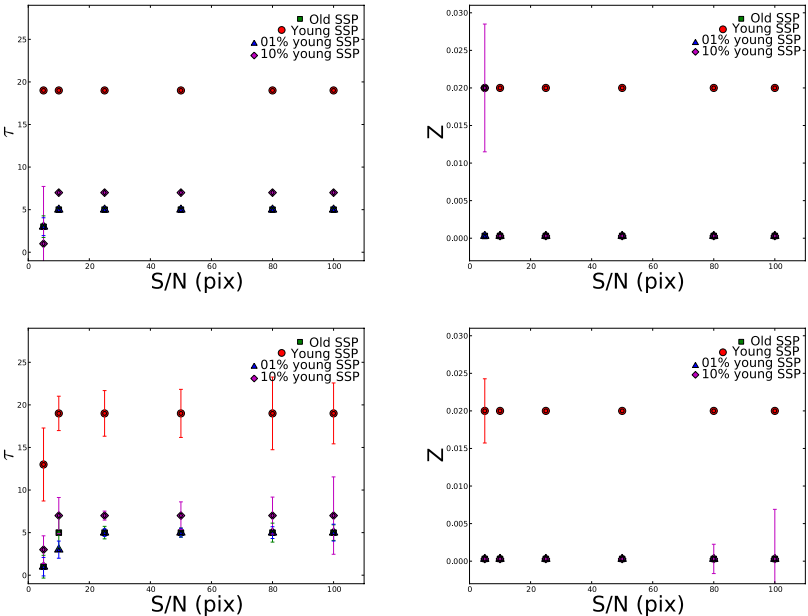

Fig. 13. Parameters of the most similar SFH spectra $(\tau-$ left panels and metallicity - right panels) obtained with the decoupling method, as a function of the $\mathrm{S} / \mathrm{N}$ in the continuum for the V600 grid. In this case, the dust attenuation has been considered known and fixed in the top panels. Results when the dust attenuation has been fitted are presented in the bottom panels. Symbols and colours are used for different underlying stellar populations of the mock galaxies, as indicated in the label.

Owing to the uncertainties obtained in the luminosityweighted age and metallicity when a grid of SSPs is considered in the decoupling method, we decided to compare our mock galaxies with spectra obtained for a given SFH (see Sect. 5.1.1). As part of the fitting procedure explained in Sect. 5.1, there is an additional option to compute the most similar spectrum to the input one from a collection of spectra. This option is independent of the global fitting to decouple the ionised gas and the stellar population, and we can feed the procedure with a different set of template spectra for this experiment. We used this option to explore which SFH spectra (Sect. 5.1.1) is more appropriate to describe our mock galaxies. In Fig. 13, we represent the $\tau$ parameter (left panels) and metallicity (right panels) for these mock galaxies, when the dust attenuation is fixed (upper panels) of fitted (lower panels). The results in both cases are similar, obtaining low values of $\tau(4<\tau<6)$ for predominantly old stellar populations and a high value $(\tau \sim 20)$ for younger stellar populations. In both cases, the metallicity is well determined, as expected since we consider two metallicity values for this experiment (see Sect. 5.1.1).

\subsection{Comparison with the SDSS data}

There are 28 galaxies observed in the sample with available SDSS spectroscopy (marked in Table 1). To compare the quality of both data sets, we extracted an integrated spectrum inside a circular aperture of $5^{\prime \prime}$ from the datacubes, centred on the intensity peak of the galaxy in the $V$-band. The extracted aperture is selected to match the aperture of the SDSS fibres, taking into account possible errors in the centroid accuracy and seeing effects in the SDSS observations, and the dithering scheme adopted in this work. We then applied the decoupling method to the spectra of both samples, deriving their physical properties. A full comparison is shown in Fig. 14. Four of the galaxies from SDSS have $\mathrm{S} / \mathrm{N}<10.0$ (per pixel, computed over the full spectra), while just one of the galaxies in our sample are below this level. As expected, there is good agreement between the values from both 

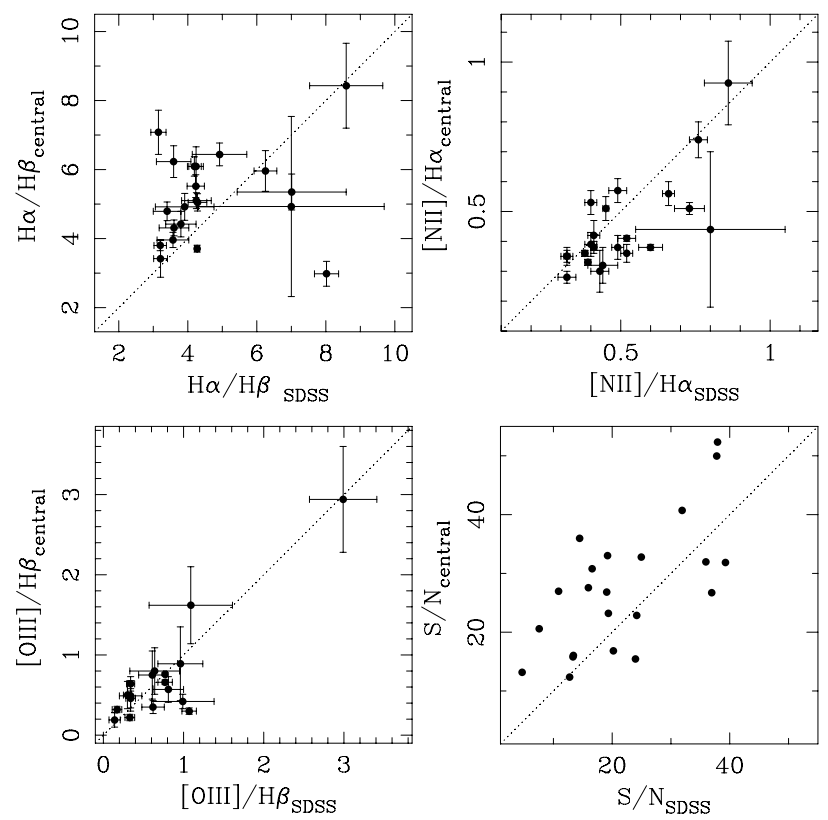

Fig. 14. Comparison of the $\mathrm{H} \alpha / \mathrm{H} \beta,\left[\mathrm{N}_{\mathrm{II}}\right] \lambda 6583 / \mathrm{H} \alpha$ and $\left[\mathrm{O}_{\mathrm{III}}\right] \lambda 5007 / \mathrm{H} \beta$ ratios, derived from central spectra for the galaxies in this sample and the SDSS spectral, using the multi-SSP fitting procedure, plus the comparison between the $\mathrm{S} / \mathrm{N}$ in both spectra.

samples, especially for the stronger, clearly de-blended emission lines, as $[\mathrm{O} \mathrm{III}] \lambda 5007 / \mathrm{H} \beta$ (determination coefficient for a least squares fits to the data $\left.r^{2}=0.79\right)$. For those galaxies with different measurements, it is possible that the SDSS fibre would not be well centred in the galaxy, and we are just comparing different regions of the galaxy. In addition, the $\mathrm{S} / \mathrm{N}$ of the galaxies observed in this work is higher in average than presented for the SDSS data.

\subsection{Aperture effects in spectroscopy}

One of the advantages of integral field spectroscopy is the possibility of integrating different combinations of the observed spectra for particular studies. For example, Rosales-Ortega et al. (2010) analysed aperture selected spectra of individual H II regions within the galaxies of the PINGS survey, and Sánchez et al. (2011b, and Viironen et al. 2011) explored the aperture effects in the galaxies of this survey, using the IFU as a large aperture spectrograph.

In this section, we analyse two aperture spectra derived for each galaxy in the sample: a central spectrum, obtained as explained in the previous section, and a $30^{\prime \prime}$ diameter aperture spectrum, which comprises most of the observed regions of each galaxy above the $5 \sigma$ detection limit. This spectrum is a good representation of the integrated properties of each galaxy. In Fig. 15, we present both aperture spectra for the subset of galaxies in Fig. 1. As can be seen, the integrated spectra have stronger emission lines than the central ones, as expected because they cover also the external parts and different components of galaxies that usually contain more ionised gas. There are also differences in the stellar continuum of both apertures for some of the galaxies, with redder colours in the central spectra than in the integrated one, which suggests that there are older stellar populations in the central part of the galaxies. For a further comparison, we applied the fitting technique presented above to both spectra, deriving the main properties for the galaxies in the sample. We present the values derived from both spectra in Fig. 16 for the main emission-line ratios. Differences between both measurements can again be seen, as expected since they are integrating different regions in the galaxies. In particular, the central aperture is mainly dominated by the bulge of galaxies, where little ionised gas is usually found. On the other hand, the integrated aperture covers most of the galaxy extent including arms, bars, and other structures in the galaxies with different gas and stellar contents.

As an example of the impact of the differences obtained when analysing both spectra, in Fig. 17 we present a classic diagnostic diagram for emission-line galaxies (e.g., Veilleux $\&$ Osterbrock 1987), consisting of the $[\mathrm{O} \mathrm{III]}] \lambda 5007 / \mathrm{H} \beta$ and $[\mathrm{N}$ II] $\lambda 6583 / \mathrm{H} \alpha$ ratios from central (blue filled squares) and integrated (green filled circles) spectra. The Kauffmann et al. (2003) (blue dashed line) and Kewley et al. (2001) (red solid line) demarcation curves usually invoked to distinguish between star forming and AGN galaxies, respectively, are also plotted. For the integrated spectra, 34 of 46 galaxies (i.e., $73.91 \%$ of the sample) have accurate emission-line ratios (with an error of $<30 \%$ ). With these data, it is found that 30 galaxies are classified as pure star-forming galaxies (88.23\% of the galaxies with accurate emission-line ratios), four galaxies are in the intermediate region $(11.76 \%)$ and there are no AGN candidates. Two galaxies in the sample show little gas $\left(F_{\mathrm{H} \alpha}<0.1 \times 10^{-16} \mathrm{cgs}\right.$ Units $)$. Based on the properties of the central spectra, just 25 (of 46) galaxies have accurate emission-line ratios (i.e., $54.35 \%$ of the sample), 14 of them are classified as star-forming galaxies $(56.00 \%)$, two galaxies $(8.00 \%)$ are AGN candidates, i.e. IC 2515, which is classified as Sy2 in the BAT catalogue ${ }^{11}$ (Cusumano et al. 2010), and CGCG 428-059, a galaxy already detected in radio and X-rays (Hardcastle et al. 2007) implying that it probably hosts an AGN. There are nine galaxies in the intermediate region $(36.00 \%)$. In particular, 2MASX J13062093+5318232, classified as an AGN in the NED database (see Table 1), appears in the intermediate region, but the error in the $\left[\mathrm{N}_{\mathrm{II}}\right] \lambda 6583 / \mathrm{H} \alpha$ ratio is $>30 \%$ and it is compatible with its classification as an AGN. Finally, in this case 18 galaxies show little gas in their central spectra.

It is clear from this comparison that important differences are found when analysing and comparing spectra extracted with different apertures over a galaxy. The 2D data from IFS surveys can be used to analyse these aperture effects (e.g., Viironen et al. 2011), which could be critical for the comparison and interpretation of galaxies at different redshifts. A large number of observed objects using this technique allows us to obtain empirical calibrations to compare in an appropriate way the values obtained in the local universe with galaxies at higher redshifts.

\subsection{Spatially resolved properties}

The main advantage of using IFS for the study of galaxies is the ability to derive spatially resolved spectroscopic properties (e.g., de Zeeuw et al. 2002; Rosales-Ortega et al. 2010; Blanc et al. 2010; Castillo-Morales et al. 2010). Using IFS, we obtain a spectrum for each position of the galaxy, and therefore the decoupling method explained before can be applied to each of the individual spectra within the obtained datacubes with enough $\mathrm{S} / \mathrm{N}$. In this way, we obtain 2D distributions (or maps) of the parameters derived by this analysis. Although it is beyond the scope of this article to analyse in detail each of them, we present

\footnotetext{
${ }^{11}$ http://www.pa.iasf.cnr.it/cgi-bin/bat/main/fonti. cgi
} 
E. Mármol-Queraltó et al.: Integral field spectroscopy of a sample of nearby galaxies. I.
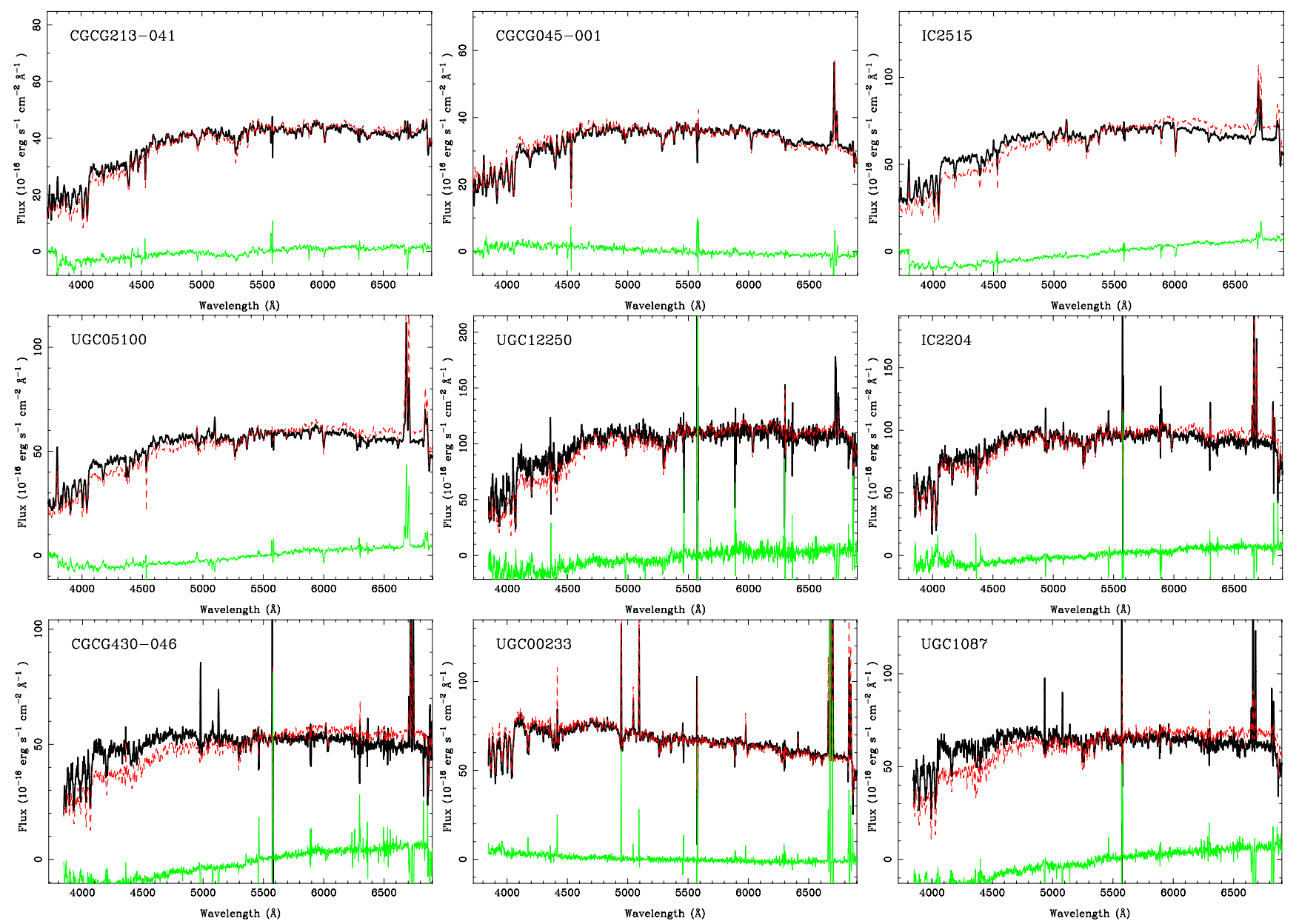

Fig. 15. Comparison of the central (red) and the integrated (black) spectra for the subset of galaxies presented in Fig. 1, normalized to the flux of the central spectrum at 5000-6000 А̊A. Differences between both spectra are plotted in green.
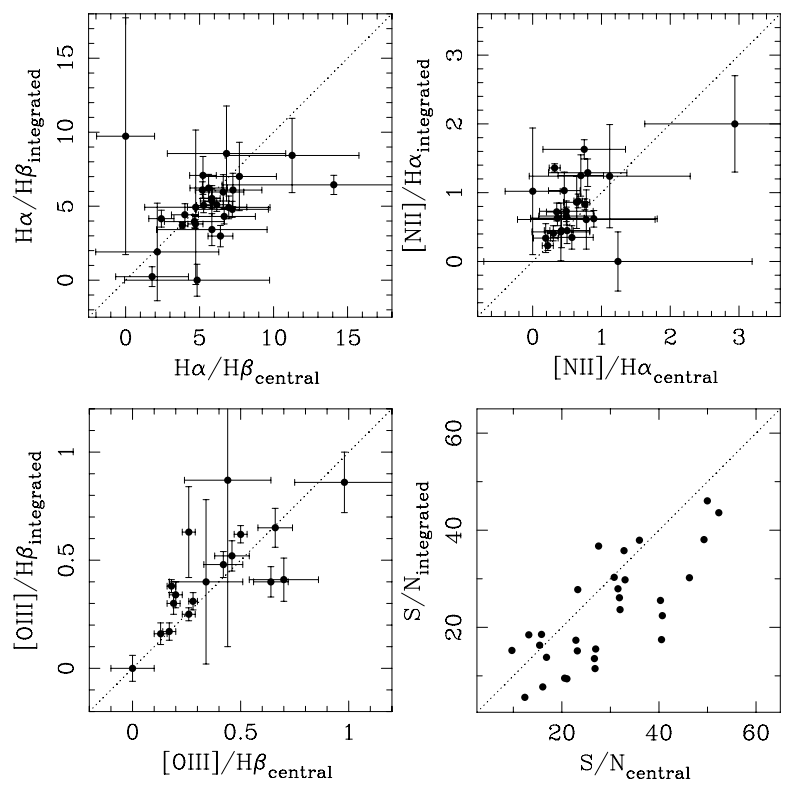

Fig. 16. Same as in Fig. 14 for the comparison of the values derived from central and integrated spectra for the galaxies in this sample.

here a few examples to show the quality of the derived maps, focusing in this case on the properties of the ionised gas.

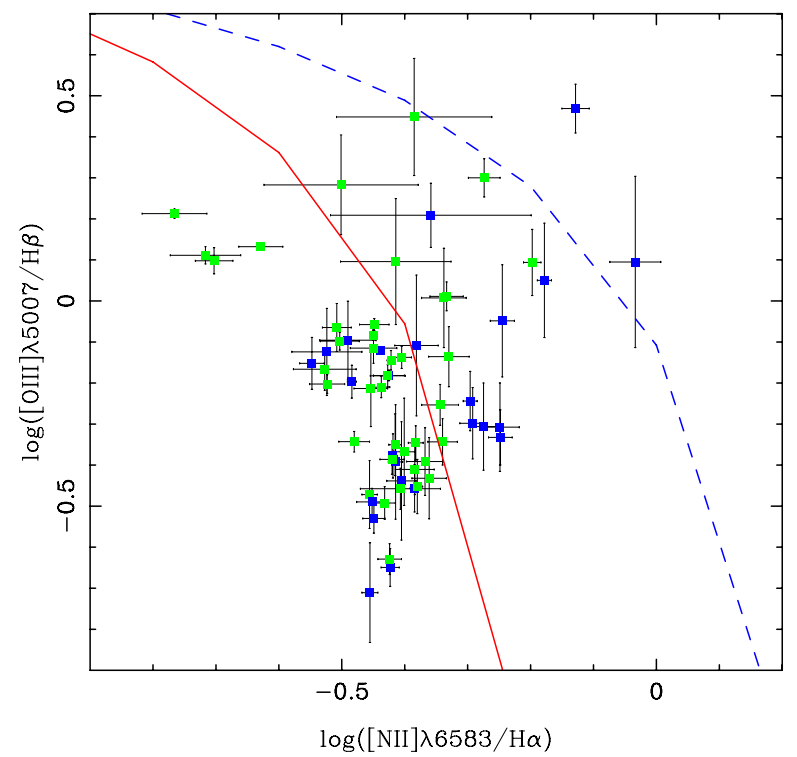

Fig. 17. The BPT diagnostic diagram for the central (blue filled squares) and integrated (green filled circles) spectra for the galaxies in the sample. The blue dashed and red solid lines separate the zones of H II-like galaxies (below the lines) and AGNs (above the lines) as defined by Kauffmann et al. (2003) and Kewley et al. (2001), respectively.

Figure 18 shows the $2 \mathrm{D}$ distribution of the $\left[\mathrm{N}_{\text {II }}\right] \lambda 6583 / \mathrm{H} \alpha$ line ratio derived from the fitting procedures described in the 

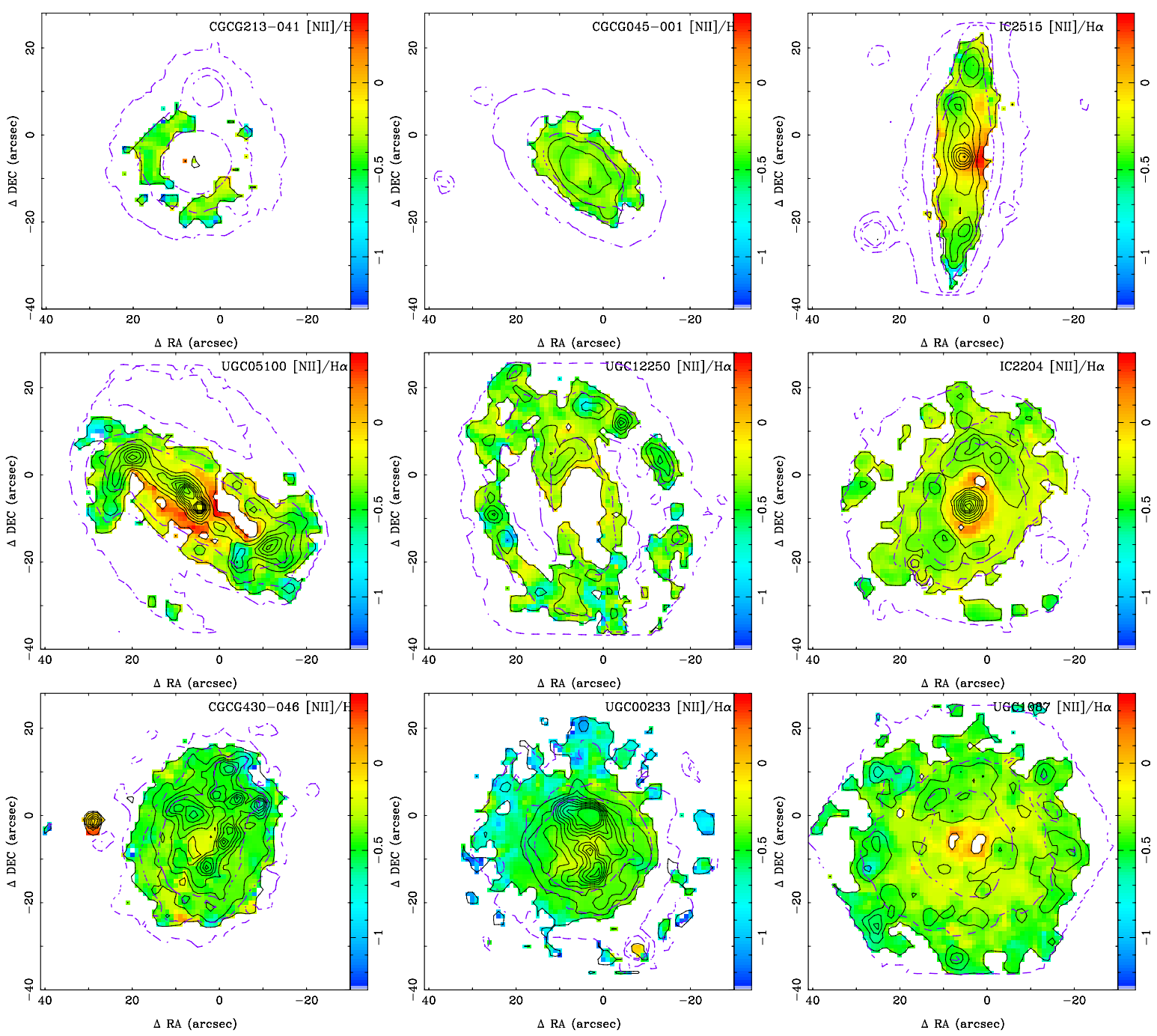

Fig. 18. Colour map of the $\left[\mathrm{N}_{\mathrm{II}}\right] \lambda 6583 / \mathrm{H} \alpha$ line ratio derived from the fitting procedures described in the text, for a subset of the observed galaxies. The black solid contours shows the $\mathrm{H} \alpha$ observed intensity, starting at $0.3 \times 10^{-16} \mathrm{erg} \mathrm{s}^{-1} \mathrm{~cm}^{-2}$, and with a step of $1 \times 10^{-16} \mathrm{erg} \mathrm{s}^{-1} \mathrm{~cm}^{-2}$ for each contour. The blue dashed contours shows the continuum intensity at the $R_{\mathrm{C}}$-band, starting at $0.03 \times 10^{-16} \mathrm{erg} \mathrm{s}^{-1} \mathrm{~cm}^{-2}$, with a step of $0.1 \times 10^{-16} \mathrm{erg} \mathrm{s}^{-1} \mathrm{~cm}^{-2}$ for each contour.

text for the subset of galaxies in Fig. 1. The blue dashed contours shows the continuum intensity at the $R_{\mathrm{C}}$-band. The black solid contours shows the $\mathrm{H} \alpha$ observed intensity, starting at $0.3 \times 10^{-16} \mathrm{erg} \mathrm{s}^{-1} \mathrm{~cm}^{-2}$. We note that the data presented in this work enable us to reach sensitivity limits of $\sim 10^{-16} \mathrm{erg} \mathrm{s}^{-1} \mathrm{~cm}^{-2}$, fainter than previous imaging surveys in the local universe (e.g. Pérez-González et al. 2003; Helmboldt et al. 2004; Meurer et al. 2006; Kennicutt et al. 2008; Karachentsev \& Kaisin 2010), which allows a more accurate determination of the SFR in the local universe. In addition, we present in Fig. 19 the velocity map of the ionised gas (from the $\mathrm{H} \alpha$ emission), derived with the previously described procedure. In most cases a clear rotational pattern is seen, mostly due to the bias towards late-type galaxies in the presented subset.

Finally, Fig. 20 shows the rotational curves along the kinematic major axis, derived from the velocity maps of $\mathrm{H} \alpha$ (Fig. 19). The colour code indicates the $[\mathrm{N} \mathrm{II}] \lambda 6583 / \mathrm{H} \alpha$ line ratio presented in Fig. 18. Values of $R_{25}$ are taken from the RC3 catalogue (see Table 1). From the sub-sample shown in Fig. 20, we can see that a $2 \mathrm{D}$ study is possible out to $\sim 1.0 R_{25}$ with the data presented in this work, although in most of cases we do not reach such a large galactic radius. This experiment shows the importance of the sample selection in accommodating the galaxies in the FoV of the PPaK instrument. Closer galaxies with larger angular sizes will have values of $R_{25}$ that are outside the FoV, while the $\mathrm{S} / \mathrm{N}$ of small (and faint) galaxies will be not enough for most 2D studies. Our study therefore illustrate the importance of the sample selection not only in terms of volume and limiting magnitude, but also the apparent size of galaxies as previous works have shown (e.g., Bershady et al. 2010a).

These figures illustrate the suitability of the considered experimental setup for the study of these spectroscopic properties within the full optical extension of the considered galaxies (characterised by the continuum emission). A detailed analysis of the resolved properties of these galaxies will be presented in forthcoming papers.

\section{Summary and conclusions}

We have presented an IFS study of nearby galaxies, exploiting the capabilities of the wide-field PPaK IFU of the PMAS spectrograph mounted on the $3.5 \mathrm{~m}$ telescope of the Calar Alto observatory (Almería, Spain). In particular, we have explored the requirements to perform a detailed study of the most relevant 
E. Mármol-Queraltó et al.: Integral field spectroscopy of a sample of nearby galaxies. I.
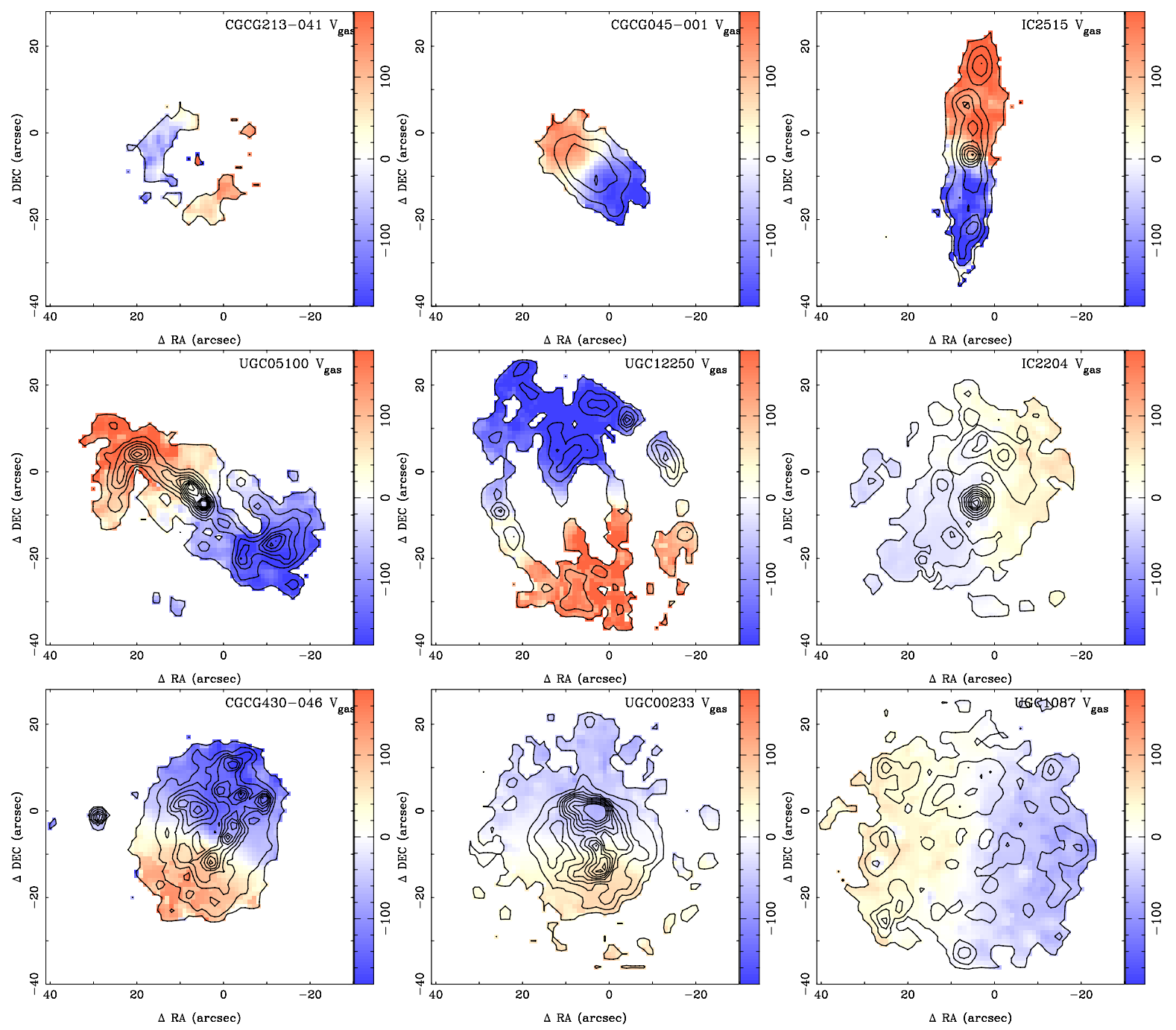

Fig. 19. For the same subset of galaxies than in previous figures, colour map of the velocity obtained from the H $\alpha$ emission, once corrected by the corresponding systemic velocity. The black solid contours shows the $\mathrm{H} \alpha$ observed intensity, starting at $0.3 \times 10^{-16} \mathrm{erg} \mathrm{s}^{-1} \mathrm{~cm}^{-2}$, and with a step of $1 \times 10^{-16} \mathrm{erg} \mathrm{s}^{-1} \mathrm{~cm}^{-2}$ for each contour, as in Fig. 18 .

spatially resolved spectroscopic properties of galaxies in the local universe across their entire optical extension, focusing on: (i) the main properties of the ionised gas; (ii) the luminosityweighted age and metallicity of the stellar population; (iii) the main kinematic properties of both the ionised gas and stellar population.

We have considered two sample selections, based on either (1) a pure redshift selection or (2) a diameter selected sample, and adopted two different instrumental setups, covering in both cases a similar FoV of $\sim 1 \operatorname{arcmin}^{2}$ and spectral range ( 3700-7050 ̊̊̊), but with different spectral resolutions of $R \sim 500$ and $R \sim 900$. We have demonstrated that it is possible to adopt a particular instrumental setup and observing strategy to observe a large sample of nearby galaxies in a reasonable amount of time (i.e., a couple of hours per target, including overheads), covering most of the optical extension of these galaxies and a wide spectroscopic optical range with $\mathrm{PPaK}$.

We have provided a detailed description of the data reduction, and we demonstrate that the large amount of data produced in this kind of observations can be handled if a dedicated automatic reduction pipeline is implemented. In addition, an automatic analysis procedure is used to handle the large number of spectra in the datacubes derived for each individual galaxy, and to obtain the their physical properties.

We have performed a set of simulations to explore the accuracy of the physical properties derived from the data. These simulations indicate that the adopted observational strategy, and in particular, the adopted resolutions and integration times are suitable for studying both the integrated and spatially resolved spectroscopic properties of these galaxies. The simulations show that the spectral range of the data is not wide enough to determine simultaneously the dust attenuation and the stellar populations, and additional information from other spectral bands, such as the UV and infrared, is needed to break the age-metallicity-dust degeneration. The recovery of the properties of the ionised gas and the kinematics can be accurately performed with the simple grid of templates used here, although the determination of the luminosity-weighted age and, especially, metallicity depends on the grid of templates used, as expected.

From the comparison of the galaxy data of this work with previously published imaging and spectroscopic data from the SDSS survey, we have shown that it is feasible (1) to obtain spectrophotometrically calibrated IFS spectra, and (2) to recover the derived spectroscopic properties equally well than with single fibre spectra. An illustrative analysis of the $2 \mathrm{D}$ properties for a 

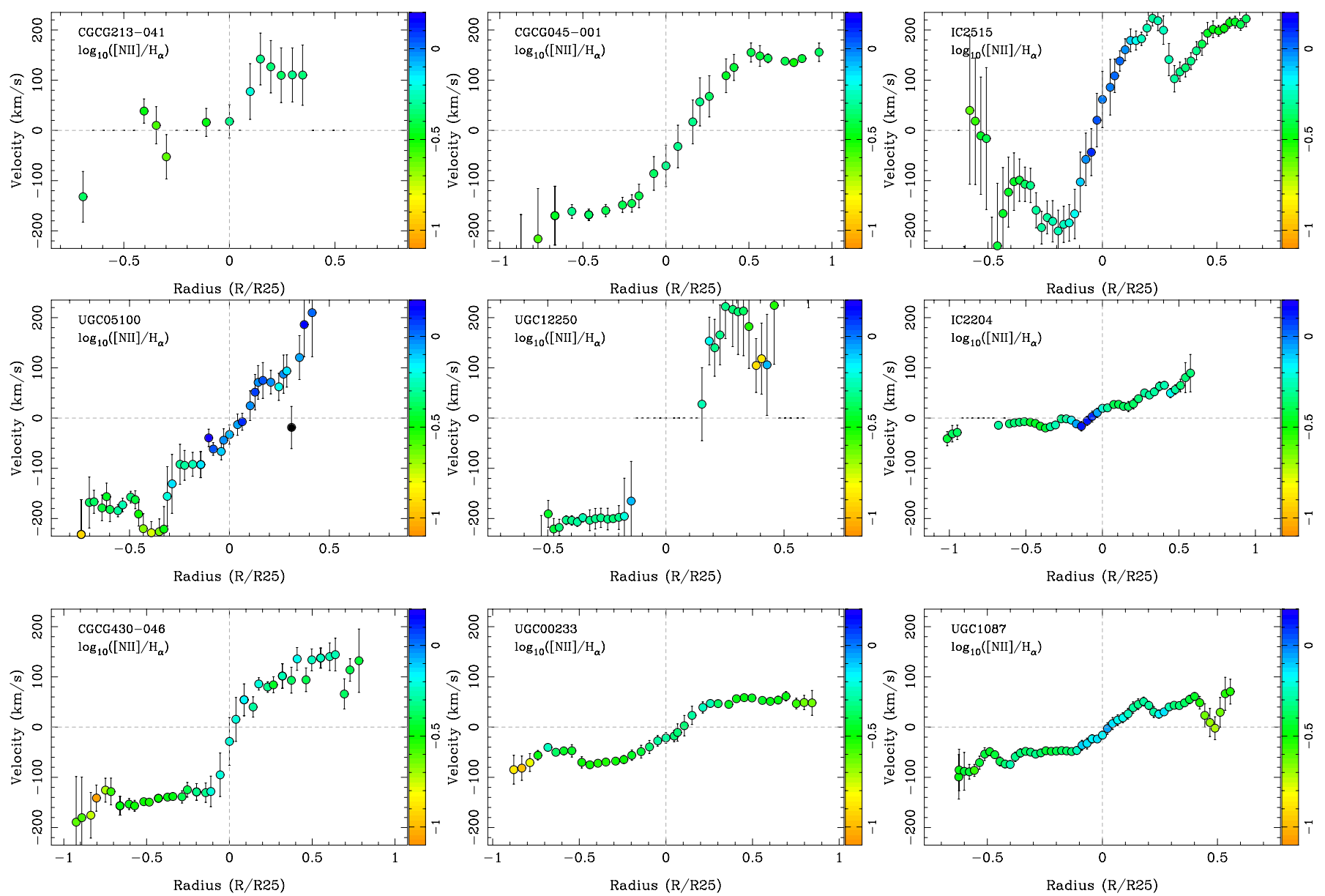

Fig. 20. Rotational curves derived from the maps shown in the previous figure along the axis of maximum rotation. The colour code indicates the $[\mathrm{N}$ II] $] \lambda 6583 / \mathrm{H} \alpha$ ratio, in logarithmic scale. The distances are normalised by the $R_{25}$ radius.

subset of galaxies of this sample indicates that it is feasible to derive the spectroscopic properties for different apertures, and to obtain accurate 2D distributions of these properties within the optical extension of these galaxies.

Finally, we wish to highlight that integral field spectroscopy constitutes a powerful tool for helping us to understand the global evolution of nearby galaxies from the analysis of their resolved physical properties. This study was part of the preparatory works for a larger IFS survey, the CALIFA survey ${ }^{12}(\sim 600$ galaxies at $0.005<z<0.030$ ), which will be an important resource for detailed studies of galaxies in the local universe.

Acknowledgements. We thank the referee, Matt Bershady, for his useful comments. We acknowledge the Viabilidad, Diseño, Acceso y Mejora funding program, ICTS-2009-10, and the Plan Nacional de Investigación y Desarrollo funding program AYA2010-22111-C03-03 and AYA-2010-10904-E, of the Spanish Ministerio de Ciencia e Innovación (MICINN), for the support given to this project. E.M.Q. acknowledges financial support from the research projects AYA2007-67752-C03-03 and AYA2010-21322-C03-02. E.M.Q., R.M. and A.G.P. thank support from Consolider-GTC and AstroMadrid S2009/ESP1496 - from the Comunidad de Madrid -. R.M. and A.G.P. acknowledge financial suppor from the project AYA2005-09413-C02-02. J.V.M. and J.I.P. are funded by the grants AYA2007-67965-C03-02 - from the Spanish MICINN and TIC114 - from the Junta de Andalucía -.

We also thank the director of CEFCA, Dr. M. Moles, for his sincere support of this project.

This paper makes use of the Sloan Digital Sky Survey data. Funding for the SDSS and SDSS-II has been provided by the Alfred P. Sloan Foundation, the Participating Institutions, the National Science Foundation, the US Department

12 http://www.caha.es/CALIFA/ of Energy, the National Aeronautics and Space Administration, the Japanese Monbukagakusho, the Max Planck Society, and the Higher Education Funding Council for England. The SDSS Web Site is http://www. sdss.org/.

The SDSS is managed by the Astrophysical Research Consortium for the Participating Institutions. The Participating Institutions are the American Museum of Natural History, Astrophysical Institute Potsdam, University of Basel, University of Cambridge, Case Western Reserve University, University of Chicago, Drexel University, Fermilab, the Institute for Advanced Study, the Japan Participation Group, Johns Hopkins University, the Joint Institute for Nuclear Astrophysics, the Kavli Institute for Particle Astrophysics and Cosmology, the Korean Scientist Group, the Chinese Academy of Sciences (LAMOST), Los Alamos National Laboratory, the Max-Planck-Institute for Astronomy (MPIA), the Max-Planck-Institute for Astrophysics (MPA), New Mexico State University, Ohio State University, University of Pittsburgh, University of Portsmouth, Princeton University, the United States Naval Observatory, and the University of Washington.

\section{References}

Bacon, R., Copin, Y., Monnet, G., et al. 2001, MNRAS, 326, 23 Bell, E. F., McIntosh, D. H., Barden, M., et al. 2004, ApJ, 600, L11 Bell, E. F., Naab, T., McIntosh, D. H., et al. 2006, ApJ, 640, 241

Bershady, M. A., Andersen, D. R., Harker, J., Ramsey, L. W., \& Verheijen, M. A. W. 2004, PASP, 116, 565

Bershady, M. A., Andersen, D. R., Verheijen, M. A. W., et al. 2005, ApJS, 156, 311

Bershady, M. A., Verheijen, M. A. W., Swaters, R. A., et al. 2010a, ApJ, 716, 198

Bershady, M. A., Verheijen, M. A. W., Westfall, K. B., et al. 2010b, ApJ, 716, 234

Blanc, G. A., Gebhardt, K., Heiderman, A., et al. 2010, ASP Conf. Ser., 432, 180 Calzetti, D. 2001, PASP, 113, 1449 
E. Mármol-Queraltó et al.: Integral field spectroscopy of a sample of nearby galaxies. I.

Cappellari, M., Emsellem, E., Krajnović, D., et al. 2011, MNRAS, 269 Cardelli, J. A., Clayton, G. C., \& Mathis, J. S. 1989, ApJ, 345, 245

Castillo-Morales, A., Gallego, J., Pérez-Gallego, J., et al. 2010, in Highlights of Spanish Astrophysics V, ed. J. M. Diego, L. J. Goicoechea, J. I. GonzálezSerrano, \& J. Gorgas, 267

Charlot, S., \& Fall, S. M. 2000, ApJ, 539, 718

Chen, Y., Lowenthal, J. D., \& Yun, M. S. 2010, ApJ, 712, 1385

Cid Fernandes, R., Mateus, A., Sodré, L., Stasińska, G., \& Gomes, J. M. 2005, MNRAS, 358, 363

Cusumano, G., La Parola, V., Segreto, A., et al. 2010, A\&A, 510, A48

de Zeeuw, P. T., Bureau, M., Emsellem, E., et al. 2002, MNRAS, 329, 513

Faber, S. M., Willmer, C. N. A., Wolf, C., et al. 2007, ApJ, 665, 265

Gavazzi, G., Bonfanti, C., Sanvito, G., Boselli, A., \& Scodeggio, M. 2002, ApJ, 576,135

Gil de Paz, A., Boissier, S., Madore, B. F., et al. 2007, ApJS, 173, 185

Hardcastle, M. J., Kraft, R. P., Worrall, D. M., et al. 2007, ApJ, 662, 166

Helmboldt, J. F., Walterbos, R. A. M., Bothun, G. D., O’Neil, K., \& de Blok,

W. J. G. 2004, ApJ, 613, 914

Hill, G. J., MacQueen, P. J., Smith, M. P., et al. 2008, SPIE Conf. Ser., 7014

Hinshaw, G., Weiland, J. L., Hill, R. S., et al. 2009, ApJS, 180, 225

Jenkins, E. B. 1987, in Interstellar Processes, ed. D. J. Hollenbach, \&

H. A. Thronson Jr., Astrophys. Space Sci. Lib., 134, 533

Jester, S., Schneider, D. P., Richards, G. T., et al. 2005, AJ, 130, 873

Karachentsev, I. D., \& Kaisin, S. S. 2010, AJ, 140, 1241

Kauffmann, G., Heckman, T. M., Tremonti, C., et al. 2003, MNRAS, 346, 1055

Kelz, A., Verheijen, M. A. W., Roth, M. M., et al. 2006, PASP, 118, 129

Kennicutt, Jr., R. C., Lee, J. C., Funes, J. G. S. J., Sakai, S., \& Akiyama, S. 2008, ApJS, 178, 247

Kewley, L. J., Dopita, M. A., Sutherland, R. S., Heisler, C. A., \& Trevena, J. 2001, ApJ, 556, 121

Koleva, M., Prugniel, P., Bouchard, A., \& Wu, Y. 2009, A\&A, 501, 1269

Lasker, B. M., Lattanzi, M. G., McLean, B. J., et al. 2008, AJ, 136, 735

MacArthur, L. A., González, J. J., \& Courteau, S. 2009, MNRAS, 395, 28
Meurer, G. R., Hanish, D. J., Ferguson, H. C., et al. 2006, ApJS, 165, 307 Monet, D. G., Levine, S. E., Canzian, B., et al. 2003, AJ, 125, 984

Muñoz-Mateos, J. C., Gil de Paz, A., Boissier, S., et al. 2007, ApJ, 658, 1006

Muñoz-Mateos, J. C., Gil de Paz, A., Boissier, S., et al. 2009, ApJ, 701, 1965

Ocvirk, P., Pichon, C., Lançon, A., \& Thiébaut, E. 2006, MNRAS, 365, 46

Oke, J. B. 1990, AJ, 99, 1621

Pérez-González, P. G., Zamorano, J., Gallego, J., Aragón-Salamanca, A., \& Gil de Paz, A. 2003, ApJ, 591, 827

Pérez-Gallego, J., Guzmán, R., Castillo-Morales, A., et al. 2010, MNRAS, 402, 1397

Rosales-Ortega, F. F., Kennicutt, R. C., Sánchez, S. F., et al. 2010, MNRAS, 405, 735

Roth, M. M., Kelz, A., Fechner, T., et al. 2005, PASP, 117, 620

Sánchez, S. F. 2004, Astron. Nachr., 325, 167

Sánchez, S. F. 2006, Astron. Nachr., 327, 850

Sánchez, S. F., Aceituno, J., Thiele, U., Pérez-Ramírez, D., \& Alves, J. 2007a, PASP, 119, 1186

Sánchez, S. F., Cardiel, N., Verheijen, M. A. W., Pedraz, S., \& Covone, G. 2007b, MNRAS, 376, 125

Sánchez, S. F., Kennicutt, R. C., Gil de Paz, A., et al. 2010 [arXiv: 1012.3002]

Sánchez, S. F., Kennicutt, R. C., Gil de Paz, A., et al. 2011a, A\&A, submitted

Sánchez, S. F., Rosales-Ortega, F. F., Kennicutt, R. C., et al. 2011b, MNRAS, 410,313

Sandage, A. 1986, A\&A, 161, 89

Sarzi, M., Falcón-Barroso, J., Davies, R. L., et al. 2006, MNRAS, 366, 1151

Skrutskie, M. F., Cutri, R. M., Stiening, R., et al. 2006, AJ, 131, 1163

Strateva, I., Ivezić, Ž., Knapp, G. R., et al. 2001, AJ, 122, 1861

Vazdekis, A., Sánchez-Blázquez, P., Falcón-Barroso, J., et al. 2010, MNRAS 404, 1639

Veilleux, S., \& Osterbrock, D. E. 1987, ApJS, 63, 295

Verheijen, M. A. W., Bershady, M. A., Andersen, D. R., et al. 2004, Astron. Nachr., 325, 151

Viironen, K., Sánchez, S. F., Mármol-Queraltó, E., et al. 2011, A\&A, submitted 